\title{
Price of anarchy for reliability-based traffic assignment and network design
}

\author{
W.Y. Szeto ${ }^{1}$ and Anny B. Wang
}

Department of Civil Engineering, The University of Hong Kong, Hong Kong, P. R. China

${ }^{1}$ Phone: (852) 28578552, Fax: (852) 25595337, Email: ceszeto@hku.hk

\begin{abstract}
This paper proposes reliability-based system-optimal traffic assignment under supply uncertainty based on the concept of the total system travel time budget, and defines the price of anarchy for the corresponding user equilibrium traffic assignment. An analytical formula for a set of the upper bounds of the price of anarchy for the equilibrium assignment to the networks with polynomial link travel time functions is derived. These bounds are proven to be independent of the network topology and demands. The formula for the minimum upper bound is also derived and can be reduced to the upper bound formula for traditional user equilibrium traffic assignment as a special case. The price of anarchy for the traditional user equilibrium network design problem with polynomial link travel time functions is also proven to be bounded by the upper bound of that for traditional user equilibrium traffic assignment of the same instance, before any link expansion. The price of anarchy for the reliability-based user equilibrium network design problem with polynomial link travel time functions is also proven to be bounded by the set of upper bounds of that for reliability-based user equilibrium traffic assignment of the same instance, before any link expansion
\end{abstract}

Keywords: reliability-based traffic assignment; network design problem; price of anarchy

\section{Introduction}

In a transportation network, road users always selfishly choose their own shortest paths to their individual destinations. This is captured by user equilibrium (UE) traffic assignment, in which every road user traveling from the same origin to the same destination bears an exactly identical travel time. In contrast to UE traffic assignment, system-optimal (SO) traffic assignment aims to minimize the total system travel time (TSTT).

Many studies have quantified the worst-case ratio of TSTT at UE to that at SO, which was first termed the "price of anarchy" (PoA) by Koutsoupias and Papadimitriou (1999). Roughgarden and Tardos (2002) mainly studied the PoA for traditional UE traffic assignment with fixed demand, in which the link travel time function is separable and link capacity constraints are not considered. Since then, four major lines of research have arisen:

- arc capacity constraints;

- demand and link travel time/cost functions;

- other equilibrium principles and multiple user classes; and

- road pricing considerations.

Correa et al. (2004) provided one example of a study belonging to the first line of research. They extended the study of Roughgarden and Tardos (2002) and examined the PoA in traditional UE traffic assignment with capacity constraints. The first line of research has 
received little attention because the results do not differ significantly from those found by Roughgarden and Tardos (2002).

The second line of research focuses on relaxing the assumption related to the time/cost functions used and considers elastic instead of fixed demand. For instance, Chau and Sim (2003) extended the study of Roughgarden and Tardos (2002) to consider the case in which link travel cost functions are non-separable but symmetric (a case that can include separable functions), and the demand of each origin-destination (O-D) pair is elastic. Perakis (2004) further considered the case in which the cost functions are both asymmetric and non-separable but the demand is fixed. Han et al. (2008a) extended their studies by considering elastic demand.

Apart from the investigation of the PoA for traditional UE traffic assignment, the third line of research considers other traffic assignment problems and more user classes. For example, Guo et al. (2010) proposed a PoA for stochastic UE traffic assignment, and Han and Yang (2008) studied the PoA for the multi-class, multi-criterion traffic equilibrium problem. In both of these studies, the travel time of a road user was usually assumed to be deterministic, which might not have reflected the actual travel time of the road user given the neglect of supply or demand stochasticity. Travelers may be risk averse and select a safer route to arrive at their individual destinations on time. It is important to extend the PoA analysis to reliability-based UE (RUE) traffic assignment to explore this issue. In addition, most RUE traffic assignment studies (e.g., Liu et al., 2009; Chen and Zhou, 2010; Sumalee and Xu, 2010) have made assumptions related to the functional form of the distribution of link travel time or capacity, such as normal distribution and log-normal distribution. However, in practice, it is not only resource consuming and expensive to obtain the data necessary to form a probability distribution function for link travel time, but it is also difficult to verify its validity in some cases because the data (such as data related to earthquake or hazardous material incidents) collected during a short period may be insufficient. This raises the need to relax these assumptions to examine the PoA for RUE traffic assignment.

The fourth line of research related to the PoA focuses on situations involving tolls (e.g., Xiao et al., 2007; Han and Yang, 2008; Han et al., 2008b). Various PoA definitions have been proposed. However, the PoA should have potential applications to many other transportation engineering problems, such as the road network design problem (NDP) (e.g., Abdulaal and Leblanc, 1979; Boyce, 1984; Yang and Bell, 1998; Meng et al., 2001; Meng and Yang, 2002; $\mathrm{Ng}$ and Waller, 2009b), because the road tolling or pricing problem can be considered a special case of the road NDP. In a road NDP, the optimal link expansion must be considered, which makes it difficult to derive the upper bound of the PoA for the NDP directly from the theoretical result of the PoA with a pricing consideration. Establishing an analytical formula for the upper bound of the PoA remains a big challenge. Furthermore, because reliability-based road network design has received much attention from many scholars (e.g., Sumalee et al., 2006, 2009; $\mathrm{Ng}$ and Waller, 2009a), it is worthwhile to examine the applications of the PoA to the reliability-based NDP.

Distribution-free reliability-based system-optimal (RSO) and RUE traffic assignment problems, analogous to SO and UE traffic assignment, are proposed and examined in this paper to define a PoA for the RUE assignment problem, where "distribution-free" means that the results are independent of the functional form of the probability distribution. In the RSO 
traffic assignment problem, users are assumed to minimize the total system travel time budget (TSTTB) (i.e., the sum of the mean TSTT plus a safety margin). In the RUE traffic assignment problem, users are assumed to select the route with the lowest users' travel time budget (TTB) involved. Both problems are assumed to have fixed demand but travel time is uncertain due to supply uncertainty. Unlike the study by Liu et al. (2009), this study considers the risk-averse behavior of travelers and the system manager. Unlike Ordoñez and Stier-Moses (2010), who focused on analyzing different formulations for reliability-based traffic assignment, this study derives an analytical form of the minimum upper bound of the PoA for the RUE traffic assignment problem that involves polynomial link travel time functions and examines the properties of the minimum upper bound. The loss of tightness of the bound is also discussed.

To mathematically analyze the PoA for NDPs, a PoA is defined in this paper for the traditional user equilibrium network design problem (UE-NDP), i.e., the continuous NDP that considers link expansion under budgetary and capacity constraints, with link additions included as a special case. The analysis is further extended to distribution-free reliability-based NDPs where the objective is to minimize the TSTTB. The two versions, i.e., the reliability-based user equilibrium network design problem (RUE-NDP) and the reliability-based system-optimal network design problem (RSO-NDP) are also defined, which correspond to the traditional UE-NDP and system-optimal network design problem (SO-NDP), respectively. RUE assignment is used to depict users' route choices in the RUE-NDP. A definition of the PoA is proposed for an RUE-NDP, and the relation between its upper bound(s) and that for the RUE traffic assignment is examined.

This paper makes the following contributions.

- It proposes distribution-free RSO and RUE traffic assignment problems that consider risk aversion and supply uncertainty, and analyzes the properties of the related problems, along with the existence and uniqueness of related solutions.

- It introduces the concept of a TSTTB.

- It proposes a definition of the PoA for RUE traffic assignment with polynomial link travel time functions and derives a set of upper bounds of the PoA.

- It proves that the set of upper bounds is independent of the demand pattern and network topology.

- It mathematically examines the properties of the minimum upper bound in terms of the problem parameters and proves that the minimum upper bound can be reduced to the bound given in the study by Roughgarden and Tardos (2002) as a special case.

- It proposes a definition of the PoA for the traditional UE-NDP with polynomial link travel time functions and proves that it is bounded by the upper bound of the PoA for traditional UE traffic assignment of the same instance, before any link expansion.

- It proposes distribution-free RSO- and RUE-NDPs using the concepts of TSTTB and TTB.

- It proposes a definition of the PoA for the RUE-NDP with polynomial link travel time functions and proves that it is bounded by a set of upper bounds of the PoA for RUE traffic assignment of the same instance, before any link expansion. 
The remainder of the paper is organized as follows. Section 2 provides a brief review of the PoA for traditional traffic assignment. Section 3 examines reliability-based traffic assignment and derives its set of upper bounds, including the upper bound of the PoA mentioned in Section 2 as a special case. Section 4 extends consideration of the PoA to the traditional NDP. It shows that the upper bound of the PoA in Section 2 is also an upper bound of the PoA for the traditional UE-NDP. Section 5 further extends the analysis of the PoA in Section 4 to reliability-based NDPs and proves that the PoA is bounded by the set of upper bounds derived in Section 3. Finally, Section 6 concludes the paper.

\section{Review of the price of anarchy for traffic assignment without uncertainty}

Consider a transportation network $G(N, A)$ with multiple O-D pairs, where $N$ denotes the set of nodes and $A$ denotes the set of links in the network. The demands between the O-D pairs are fixed. The following notations (Table 1) are defined for ease of discussion.

Table 1 Notations

\begin{tabular}{|l|l|}
\hline$\Phi_{+}$ & Set of positive integers \\
\hline$R S$ & Set of O-D pairs \\
\hline$P_{r s}$ & Set of paths between O-D pair $r s \in R S$ \\
\hline$d_{r s}$ & Travel demand between O-D pair $r s \in R S$ \\
\hline$v_{a}$ & Flow on link $a \in A$ \\
\hline$t_{a}()$ or $t_{a}$ & Travel time function of link $a \in A$ \\
\hline$t_{a}^{0}$ & Free-flow travel time for link $a \in A$ \\
\hline$c_{a}$ & (Initial) capacity of link $a \in A$ \\
\hline$\delta_{p, r s}^{a}$ & $\begin{array}{l}\text { Indicator variable that equals } 1 \text { if link } a \in A \text { is on path } p \in P_{r s} \\
\text { between O-D pair } r s \in R S \text {, and } 0 \text { otherwise }\end{array}$ \\
\hline$t_{p, r s}$ & Travel time on path $p \in P_{r s}$ between O-D pair $r s \in R S$ \\
\hline$b_{p, r s}$ & TTB on path $p \in P_{r s}$ between O-D pair $r s \in R S$ \\
\hline$f_{p, r s}$ & Flow on path $p \in P_{r s}$ between O-D pair $r s \in R S$ \\
\hline$\tilde{\pi}_{r s}$ & Minimum travel time between O-D pair $r s \in R S$ \\
\hline$\pi_{r s}$ & TTB between O-D pair $r s \in R S$ \\
\hline
\end{tabular}




\begin{tabular}{|c|c|}
\hline$\sigma(\cdot)$ & Standard deviation of \\
\hline f & Path flow vector, $\mathbf{f}=\left(f_{p, r s}\right)_{p \in P_{r s}, r s \in R S}$ \\
\hline $\mathbf{v}$ & Link flow vector, $\mathbf{v}=\left(v_{a}\right)_{a \in A}$ \\
\hline $\mathbf{v}^{U E}$ & UE link flow vector $\mathbf{v}^{U E}=\left(v_{a}^{U E}\right)_{a \in A}$ \\
\hline $\mathbf{v}^{\text {SO }}$ & SO link flow vector $\mathbf{v}^{S O}=\left(v_{a}^{S O}\right)_{a \in A}$ \\
\hline $\mathbf{D}$ & Demand vector $\mathbf{D}=\left(d_{r s}\right)_{r s \hat{~} R S}$ \\
\hline $\mathbf{t}$ & Vector of polynomial link travel time functions $\mathbf{t}=\left(t_{a}\right)_{a \hat{\imath} \mathrm{A}}$ \\
\hline$\sigma$ & Covariance matrix of link travel times $\sigma=\left(\operatorname{Cov}\left(T_{a}, T_{b}\right)\right)_{a, b \in A}$ \\
\hline$\Omega_{f}$ & $\begin{array}{l}\text { Set of feasible path flow vectors } \\
\Omega_{f}=\left\{\mathbf{f} \mid f_{p, r s} \geq 0, \sum_{p \in P_{r s}} f_{p, r s}=d_{r s}, \forall p \in P_{r s}, \forall r s \in R S\right\}\end{array}$ \\
\hline$\Omega_{v}$ & $\begin{array}{l}\text { Set of feasible link flow vectors } \\
\Omega_{v}=\left\{\mathbf{v} \mid \mathbf{f} \in \Omega_{f}, v_{a}=\sum_{r s \in R S} \sum_{p \in P_{r s}} f_{p, r s} \delta_{p, r s}^{a}, \forall a \in A\right\}\end{array}$ \\
\hline
\end{tabular}

According to the preceding setting, two traditional traffic assignment problems have been proposed in the literature: SO and UE. The SO traffic assignment problem assigns traffic based on the second principle of Wardrop (1952), which states that the average journey time is minimal. This problem determines a link flow pattern to minimize the TSTT and can be mathematically expressed as

$$
\mathrm{SO}: \min _{\mathbf{v} \in \Omega_{v}} \operatorname{TSTT}(\mathbf{v})=\sum_{a \in A} t_{a}\left(v_{a}\right) v_{a},
$$

where $T S T T(\mathbf{v})$ denotes the TSTT at the link flow solution $\mathbf{v}$.

The UE problem assigns traffic according to Wardrop's first principle, which implies that the travel times on used routes are not greater than the travel times on unused routes between the same O-D pair. The UE conditions for route flows can be written as follows:

$$
\mathrm{UE}: f_{p, r s}\left(t_{p, r s}-\tilde{\pi}_{r s}\right)=0,\left(t_{p, r s}-\tilde{\pi}_{r s}\right) \geq 0, \forall p \in P_{r s}, \forall r s \in R S \text {, }
$$

where $t_{p, r s}=\sum_{a \in A} t_{a}\left(v_{a}\right) \delta_{p, r s}^{a}$. The UE problem can be formulated into the following variational inequality: to determine $\mathbf{v}^{U E}$ such that

$$
\sum_{a \in A}\left(v_{a}-v_{a}^{U E}\right) t_{a}\left(v_{a}^{U E}\right) \geq 0, \forall \mathbf{v} \in \Omega_{v} .
$$

The TSTT at UE is generally higher than that at SO. To measure the relative increase in the TSTT at UE to that at SO, the PoA of an instance $(G, \mathbf{D}, \mathbf{t})$ is introduced and defined as 


$$
\rho(G, \mathbf{D}, \mathbf{t})=\frac{T S T T\left(\mathbf{v}^{U E}\right)}{\operatorname{TSTT}\left(\mathbf{v}^{S O}\right)} .
$$

If $I_{n}$ is the set of all instances with polynomial travel time functions with the highest degree $n \hat{\imath} \Phi_{+}$, then the PoA of $I_{n}$ defined as $\rho\left(I_{n}\right)=\sup _{(G, \mathbf{D}, \mathbf{t}) \in I_{n}} \rho(G, \mathbf{D}, \mathbf{t})$. Roughgarden (2003) proved that the following is true: $\rho\left(I_{n}\right) \leq \rho^{\prime}$, where

$$
\rho^{\prime}=\left(1-n(n+1)^{-\frac{n+1}{n}}\right)^{-1} .
$$

However, Roughgarden's study was based on the assumption of no uncertainty in the network, which is not the case in reality.

\section{Price of anarchy for reliability-based traffic assignment}

\subsection{Supply and travel time uncertainty}

Network supply uncertainty refers to the variations in link capacities or free-flow travel times caused by disruptions to the network such as accidents, road maintenance, weather, and traffic management and control (Lo et al., 2006; Chen and Zhou, 2010). The classical approach to capturing supply uncertainty in a network model is to assume that each link capacity or free-flow travel time follows a certain distribution and that the link travel time distribution can be derived. Because a link capacity or free-flow travel time variation leads to a variation in the link travel time, an alternative approach is to focus directly on modeling travel time variations due to supply uncertainty (e.g., Ng and Waller, 2009; Chen and Zhou, 2010).

Denote the random variable $T_{a}$ as the actual travel time on link $a$. It is defined as follows:

$$
T_{a}=t_{a}\left(v_{a}\right)+x_{a}, " a ? \text { A, }
$$

where $t_{a}\left(v_{a}\right)$ is a deterministic travel time on link $a$ predicted by its link performance function, and $\xi_{a}$ is the random error between the actual and predicted link travel times. The expectation of the error term is null, i.e., $E\left(\xi_{a}\right)=0$ (e.g., $\mathrm{Ng}$ and Waller, 2009a), implying that $E\left(T_{a}\right)=t_{a}, \forall a \in A$. The random error $\xi_{a}$ captures the effect of supply uncertainty on the travel time on link $a$. The error may correlate to errors on other links, but it is independent of the predicted travel times of any of the other links. To avoid loss of generality, no specific functional form of the distribution of $\xi_{a}$ is assumed. For a network without supply uncertainty, $\xi_{a}$ is simply zero.

To facilitate the examination, the following assumptions are introduced.

A1. The standard deviation of the error term, $\sigma\left(\xi_{a}\right)$, is finite. Thus, $\sigma\left(T_{a}\right)$ is also finite and equals $\sigma\left(\xi_{a}\right)$. The random errors of two links $\xi_{i}$ and $\xi_{j}, i, j \in A$ (and also the two travel times) may be correlated. Their correlation is expressed as $\operatorname{Cov}\left(\xi_{i}, \xi_{j}\right)$ and equals $\operatorname{Cov}\left(T_{i}, T_{j}\right)$. 
A2. The predicted link travel time function $t_{a}\left(v_{a}\right)$ takes the following polynomial form:

$$
t_{a}\left(v_{a}\right)=\sum_{m=0}^{n_{a}} k_{a m} v_{a}{ }^{m}, \quad k_{a m} \geq 0, m=0,1 \ldots, n_{a}, k_{a 0}>0, k_{a n_{a}}>0,
$$

where $k_{a m}$ denotes the coefficient associated with $v_{a}{ }^{m}$, and $n_{a}$ is the highest degree of the polynomial function.

Remark 1: The polynomial function (7) includes BPR-type functions as a special case, with the form $t_{a}=t_{a}^{0}+k_{a n_{a}} v_{a}{ }^{n_{a}}$, where the constant term $t_{a}^{0}$ is the minimum free-flow travel time on link $a$ under supply uncertainty and free-flow travel time when there is no uncertainty. $n_{a} \hat{\imath} \Phi_{+}$and $k_{a n_{a}}=t_{a}^{0} \beta_{a} c_{a}^{-n_{a}}$. The constant $c_{a}$ is the (maximum) capacity and $\beta_{a}>0$ is a coefficient associated with the link flow in the BPR function.

Remark 2: The coefficient $k_{a 0}$ is larger than zero because it represents free flow travel time.

Remark 3: Yang et al. (2008) examined the PoA of selfish routing with atomic Cournot-Nash players, each controlling a strictly positive splittable amount of flow. They used the link travel time function with the form $t_{a}\left(v_{a}\right)=k_{a 0}+k_{a n_{a}} v_{a}{ }^{n_{a}}, k_{a 0}>0, k_{a n_{a}}>0$, $n_{a} \hat{\imath} \Phi_{+}$, a special case of (7).

Remark 4: The travel time function (7) is positive, continuous, separable, differentiable, monotone, and convex. If the degree of the function is larger than one, it is strictly convex.

Under assumption A1, $\sigma\left(T_{a}\right)$ and $\operatorname{Cov}\left(T_{i}, T_{j}\right)$ are both bounded, as stated in Propositions 1 and 2.

Proposition 1. Denote a non-negative multiplier $\varepsilon_{a}$, $a \in A$. Let $\varepsilon_{a}$ be $\sigma\left(T_{a}\right) / k_{a 0}$. Then, the following must be true:

$$
\sigma\left(T_{a}\right) \leq \varepsilon_{a} t_{a}, \forall a \in A
$$

Proof. Substituting $\varepsilon_{a}=\sigma\left(T_{a}\right) / k_{a 0}$ into $\varepsilon_{a} t_{a}$, the following is obtained: $\varepsilon_{a} t_{a}=\sigma\left(T_{a}\right) \frac{t_{a}}{k_{a 0}}$. The term $\sigma\left(T_{a}\right) \frac{t_{a}}{k_{a 0}}$ is not less than $\sigma\left(T_{a}\right)$ because the mean travel time $t_{a}$ must not be less than the free-flow travel time $k_{a 0}$ based on assumption A2. This completes the proof. "

Proposition 2. $\varepsilon_{a}$ is the multiplier defined in Proposition 1. Then, the travel time covariance between any two links $i$ and $j$ is bounded by $\varepsilon_{i} t_{i} \varepsilon_{j} t_{j}, \forall i, j \in A, i \neq j$ :

$$
\operatorname{Cov}\left(T_{i}, T_{j}\right) \leq \varepsilon_{i} t_{i} \varepsilon_{j} t_{j}, \forall i, j \in A, i \neq j .
$$

Proof. According to the Cauchy-Schwarz Inequality, the following can be obtained: 


$$
\left|\operatorname{Cov}\left(T_{i}, T_{j}\right)\right| \leq \sigma\left(T_{i}\right) \sigma\left(T_{j}\right), \forall i, j \in A, i \neq j .
$$

According to Proposition 1, $\sigma\left(T_{i}\right) \leq \varepsilon_{i} t_{i}$ and $\sigma\left(T_{j}\right) \leq \varepsilon_{j} t_{j}$. Hence, the right side of (10) is not greater than a non-negative number $\varepsilon_{i} t_{i} \varepsilon_{j} t_{j}$, which is also true when the absolute value sign is removed. This completes the proof. "

\section{Example 1: Illustration for Propositions 1 and 2}

The example network has one O-D pair: (A, C). The demand level is 1 . The travel time functions for the three links and their standard deviations are listed in Table 2. The travel time covariances are $\operatorname{Cov}\left(T_{1}, T_{2}\right)=0 ; \operatorname{Cov}\left(T_{1}, T_{3}\right)=0.00035 ;$ and $\operatorname{Cov}\left(T_{2}, T_{3}\right)=0$.

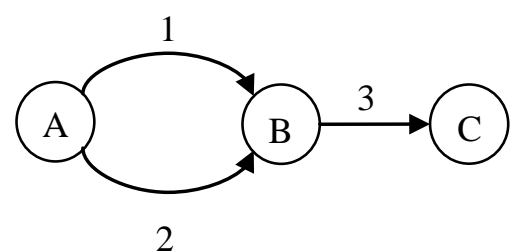

Figure 1 Example network
Table 2 Link performance functions and standard deviations

\begin{tabular}{|l|l|l|}
\hline Link & \multicolumn{1}{|c|}{$t_{a}\left(v_{a}\right)$} & \multicolumn{1}{c|}{$\sigma\left(T_{a}\right)$} \\
\hline 1 & $1+5 v_{1}$ & 0.07 \\
\hline 2 & 6.07 & 0 \\
\hline 3 & $0.1+0.1 v_{3}$ & 0.005 \\
\hline
\end{tabular}

According to Proposition 1, $\varepsilon_{a}$ equals $\sigma\left(T_{a}\right) / k_{a 0}$. Hence, $\varepsilon_{1}=0.07, \varepsilon_{2}=0$, and $\varepsilon_{3}=0.05$.

Furthermore, the covariances are bounded under any feasible flow patterns according to Proposition 2.

Clearly, $\operatorname{Cov}\left(T_{1}, T_{2}\right) \leq \varepsilon_{1} t_{1} \varepsilon_{2} t_{2}$ and $\operatorname{Cov}\left(T_{2}, T_{3}\right) \leq \varepsilon_{2} t_{2} \varepsilon_{3} t_{3}$ hold when $\varepsilon_{2}=0$. By definition, $v_{3}=1$ as this is the only link connected to C. Hence, $t_{3}=0.1+0.1(1)=0.2$. The minimum travel time on link 1 occurs when $v_{1}=0$. Under this worst case, $t_{1}=1$ and $\varepsilon_{1} t_{1} \varepsilon_{3} t_{3}=0.0007 \geq \operatorname{Cov}\left(T_{1}, T_{3}\right)=0.00035$. More flow on link 1 leads to a larger left side. Therefore, condition (9) is satisfied for any feasible flow pattern."

\subsection{The mean and standard deviation of path and total system travel times}

Denote the random variable $T_{p, r s}$ as the actual travel time on path $p \in P_{r s}$ between O-D pair $r s \in R S$, whose expression is

$$
T_{p, r s}=\sum_{a \in A} T_{a} \delta_{p, r s}^{a}, \forall p \in P_{r s}, \forall r s \in R S .
$$

Because the link flows are deterministic, the expectation and standard deviation of $T_{p, r s}$ can, respectively, be obtained by

$$
\begin{gathered}
E\left(T_{p, r s}\right)=\sum_{a \in A} E\left(T_{a}\right) \delta_{p, r s}^{a}=\sum_{a \in A} t_{a} \delta_{p, r s}^{a}, \forall p \in P_{r s}, \forall r s \in R S, \text { and } \\
\sigma\left(T_{p, r s}\right)=\sqrt{\sum_{a \in A} \sigma^{2}\left(T_{a}\right) \delta_{p, r s}^{a}+\sum_{i, j \in A, i \neq j} \operatorname{Cov}\left(T_{i}, T_{j}\right) \delta_{p, r s}^{i} \delta_{p, r s}^{j}}, \forall p \in P_{r s}, \forall r s \in R S .
\end{gathered}
$$

The total system travel time, which is a compound random variable, can be expressed as 


$$
\text { TSTT }=\sum_{a \in A} T_{a} v_{a} .
$$

Because the link flows are deterministic, the expectation and standard deviation of the TSTT can, respectively, be written as

$$
\begin{gathered}
E(\text { TSTT })=\sum_{a \in A} E\left(T_{a}\right) v_{a}=\sum_{a \in A} t_{a} v_{a} \text { and } \\
\sigma(\text { TSTT })=\sqrt{\sum_{a \in A} \sigma^{2}\left(T_{a}\right) v_{a}{ }^{2}+\sum_{i, j \in A, i \neq j} v_{i} v_{j} \operatorname{Cov}\left(T_{i}, T_{j}\right)} .
\end{gathered}
$$

\subsection{Path travel time budget and reliability-based user equilibrium assignment}

When faced with travel time uncertainties, travelers often depart early and reserve extra time for their trips to avoid late arrival. The concept of TTB was proposed to capture this behavior in traffic assignment (see Lo et al., 2006; Siu and Lo, 2008; Ordoñez and Stier-Moses, 2010).

A TTB is defined as the mean trip travel time plus the safety margin. The safety margin represents the extra time reserved by travelers. It is often defined as the standard deviation of the trip travel time multiplied by a parameter that represents their degree of risk aversion and relates to their probability of being late. For instance, if a traveler's path travel time follows a normal distribution and the value of the parameter equals 1.65 , then the probability of the traveler arriving at his/her destination on time is $95 \%$. However, if the value equals zero instead, then the probability is only $50 \%$. This concept can be used in traffic assignment that considers supply uncertainty (e.g., Lo et al., 2006) or demand uncertainty (e.g., Shao et al., 2006; Lam et al., 2008).

Regardless of the functional form of the distribution of uncertainty, the larger is the TTB compared with the mean travel time, the larger is the safety margin, the more extra time is reserved by travelers to avoid being late, and the more risk averse are the travelers.

The concept and behavioral principle used by Lo et al. (2006) for traffic assignment is adopted in this study. The path TTB $b_{p, r s}$ is formulated as follows:

$$
\begin{aligned}
b_{p, r s} & =E\left(T_{p, r s}\right)+\sigma\left(T_{p, r s}\right) \\
& =\sum_{a \in A} t_{a} \delta_{p, r s}^{a}+\lambda \sqrt{\sum_{a \in A} \sigma^{2}\left(T_{a}\right) \delta_{p, r s}^{a}+\sum_{i, j \in A, i \neq j} \operatorname{Cov}\left(T_{i}, T_{j}\right) \delta_{p, r s}^{i} \delta_{p, r s}^{j}}, \\
\forall p & \in P_{r s}, \forall r s \in R S,
\end{aligned}
$$

where $\lambda$ is a non-negative parameter called the traveler's risk aversion factor. It measures the extent to which a traveler is risk averse and how he/she makes tradeoffs between the mean and standard deviation of trip travel time. A large $\lambda$ value implies that the traveler places more weight on the effect of uncertainty, as reflected by the standard deviation. The $\lambda$ value should be calibrated from transportation survey data (see Lo et al., 2006; Szeto et al., 2011) and depends on the purpose of the trip, as demonstrated by Lo et al. (2006) using their survey data. In this paper, it is assumed that travelers are homogeneous, and as such, the $\lambda$ values for all users are identical. However, unlike other studies, this paper does not require any assumption related to the functional form of link travel time distribution.

Similar to Lo et al. (2006), this paper postulates that travelers acquire the expectations and variabilities of path travel times based on their past experiences and factor these variabilities into their route choice considerations in the form of a TTB. Moreover, all travelers want to minimize their TTBs. An equilibrium is reached only if the TTBs of all used routes are not higher than those of unused routes. This equilibrium is referred to as RUE, and the flow pattern at RUE satisfies the following: 


$$
f_{p, r s}^{R U E}\left(b_{p, r s}^{R U E}-\pi_{r s}\right)=0,\left(b_{p, r s}^{R U E}-\pi_{r s}\right) \geq 0, \quad \forall p \in P_{r s}, \forall r s \in R S .
$$

The constraints of RUE assignment are the same as those of traditional UE assignment except that the (minimum) path travel time is replaced by a (minimum) path TTB. Note that the travel time budget is expressed in terms of means, standard deviations, and covariances of link travel times for the purpose of the deviation of upper bounds of the PoA later. From the travelers' perspective, the information of the covariances of link travel times are not needed in making route choice decisions when the means and standard deviations of path travel times are known.

\section{Example 2: Reliability-based user equilibrium example}

The network setting in Example 1 is used here. In Figure 1, the upper and lower paths between O-D pair (A, C) are referred to as paths 1 and 2, respectively. Assume $\lambda$ equals 1 .

Based on (12), (13), and (17), the two path TTBs in terms of link flows are:

$b_{1, A C}=\left(1+5 v_{1}\right)+\left(0.1+0.1 v_{3}\right)+1 \cdot \sqrt{\left(0.07^{2}+0.005^{2}\right)+2 \cdot 0.00035}=1.175+5 v_{1}+0.1 v_{3}$, and $b_{2, A C}=6.175+0.1 v_{3}$.

According to the flow conservation and the relation between link and path flows, $v_{1}=f_{1, A C}$ and $v_{3}=f_{1, A C}+f_{2, A C}=d_{A C}=1$. Hence, $b_{1, A C}=1.275+5 f_{1, A C}$ and $b_{2, A C}=6.275$.

When all users choose path 1 (i.e., $f_{1, A C}=1$ and $f_{2, A C}=0$ ), $b_{1, A C}=6.275 \leq b_{2, A C}$ and the RUE condition (18) is satisfied. Hence, this flow pattern is at RUE and the minimum TTB is 6.275."

To solve for a route flow solution that satisfies condition (18) for general cases using existing projection methods (e.g., Han and Lo, 2002) and to analyze the existence and uniqueness of solutions, the following variational inequality (VI) problem is proposed.

Proposition 3. The RUE assignment problem of an instance $(G, \mathbf{D}, \mathbf{t}, \boldsymbol{\sigma})$ can be expressed as the following VI problem: to determine $\mathbf{f}^{R U E}=\left(f_{p, r s}^{R U E}\right)_{p \in P_{r s}, r s \in R S} \in \Omega_{f}$ such that

$$
\sum_{r s \in R S} \sum_{p \in P_{r s}}\left(\tilde{f}_{p, r s}-f_{p, r s}^{R U E}\right) b_{p, r s}^{R U E} \geq 0, \tilde{\mathbf{f}}=\left(\tilde{f}_{p, r s}\right)_{p \in P_{r s}, r s \in R S} \in \Omega_{f},
$$

where $b_{p, r s}^{R U E}$ is the TTB at RUE.

Proof. This proof follows that provided in a study by Smith (1979) by replacing the path travel time with a path TTB. "

The solution set of VI (19) is convex because the intersection of the nonnegative orthant and linear constraint is a convex set. The solution set is bounded because the route flows cannot be larger than the corresponding demand. The set is also closed because it includes the feasible solutions on the boundary. Hence, the set is compact. Because the link travel time functions are continuous with respect to path flows, the mapping function $b_{p, r s}$ is also continuous with respect to path flows. Based on Theorem 1.4 provided in a study by Nagurney (1993), there exists an optimal path flow solution to VI (19).

In terms of solution uniqueness, it is well known that even the traditional UE assignment problem may have multiple path flow solutions. Hence, it is expected that an optimal solution 
to VI (19) may not be unique unless the mapping function is strictly monotone ${ }^{1}$ with respect to path flows.

If $\left(t_{a}\right)_{a \uparrow A}$ is strictly monotone with respect to $\mathbf{v}$, then both the optimal link flow solution and $\left(b_{p, r s}^{R U E}\right)_{p \nmid P_{r s}, r s R S}$ are unique (see Appendix A). If $\left(t_{a}\right)_{a \hat{\uparrow} A}$ is only monotone with respect to $\mathbf{v}$, then $\left(b_{p, r s}^{R U E}\right)_{p \nmid p_{r s}, r s R S}$ is unique (see also Appendix A).

Similar to robust optimization-based traffic assignment (e.g., Sun et al., 2014), reliability-based traffic assignment is distribution free in the sense that knowledge of the distribution of each link travel time is not required. Instead, only partial information related to the distribution is required for the RUE assignment problem: the mean, standard deviation, and covariance of the random variables, i.e., the link travel times. This requirement contrasts with that of robust optimization-based traffic assignment, which requires the modeler to explicitly define an uncertainty set to be, for example, the box or ellipsoidal uncertainty set, for each random variable.

The VI problem (19) is path based (i.e., using path flows as decision variables) and cannot be reformulated as link based (i.e., using link flows as decision variables) as in a classical UE assignment in general because

- there are correlations among link travel times, and

- even if there are no correlations among link travel times, path TTBs are not link separable.

These properties make derivation of the upper bounds of the PoA for RUE assignment difficult, and we are unable to follow the derivation for the classical UE assignment directly. The two exceptions are that when there is no supply uncertainty (i.e., link travel time variances and covariances are null) or when $\lambda$ equals zero, the VI problem (19) can be reduced to a classical UE assignment, which can be expressed as a link-based VI problem.

\subsection{Total system travel time budget and reliability-based system optimum assignment}

Without considering uncertainty in traffic assignment, the classical system performance measure is the TSTT. The TSTT under SO traffic assignment has the best system performance because the TSTT has been minimized. For a network with supply uncertainty, it is essential for the system manager to be risk averse to account for the TSTT variation and make an appropriate tradeoff between the mean performance and performance variation. The TSTT variation may be large such that the mean TSTT is much smaller than the actual TSTT and is unsuitable for reflecting the actual, very bad performance. To address this issue, the concept of the TSTTB is introduced and defined as:

$$
\begin{aligned}
\text { TSTTB }_{R} & =E(T S T T)+R \cdot \sigma(T S T T) \\
& =\sum_{a \in A} t_{a} v_{a}+R \sqrt{\sum_{a \in A} \sigma^{2}\left(T_{a}\right) v_{a}{ }^{2}+\sum_{i, j \in A, i \neq j} v_{i} v_{j} \operatorname{Cov}\left(T_{i}, T_{j}\right)},
\end{aligned}
$$

where $R$ is a non-negative parameter that represents the degree of the system manager's risk aversion, and the TSTTB is the manager's largest acceptable TSTT. Similar to the travelers' risk aversion factor, a larger $R$ value means that the system manager is more risk averse, the safety margin is larger, and the probability of the actual TSTT being greater than the TSTTB is smaller. In the special case where $R=1$, the performance measure TSTTB is simply the

\footnotetext{
${ }^{1}$ A vector function $\mathbf{F}: C \rightarrow \square^{m}$ is strictly monotone on a non-empty set $C$ if for all vectors

$\mathbf{x}, \mathbf{y} \in C, \mathbf{x} \neq \mathbf{y}, \quad(\mathbf{y}-\mathbf{x})^{T}(\mathbf{F}(\mathbf{y})-\mathbf{F}(\mathbf{x}))>0$.
} 
sum of the mean and standard deviation of the TSTT (e.g., Ng and Waller, 2009a), and the safety margin exactly equals the standard deviation. When $R=0$, the manager ignores the variation of the TSTT, and only the mean TSTT is used as a system performance measure. This concept is different from the current TTB concept in the sense that whereas the TSTTB is a system measure considered by system managers, the path TTB is a route measure considered by travelers.

The definition of the TSTTB is consistent with the definition of the total TTB proposed by Chen et al. (2007). It is simply formulated using a different approach. Chen et al. (2007) defined the total TTB using the chance constraint approach without explicitly using the mean and variance of the TSTT, both of which are used to define the TSTTB in this paper. The formulation approach taken in this paper has the following advantages. First, the concept of the TSTTB is easy to understand as its mathematical structure is similar to that of the TTB. Second, this paper lends new insights into the TSTTB, including an interpretation of the $R$ value, the concept of the system manager's safety margin, and the concept of the largest acceptable TSTT.

Analogous to SO assignment, the TSTTB is used to define RSO assignment. It can be mathematically expressed as the following minimization program:

$$
\min _{\mathbf{v} \in \Omega_{v}} \operatorname{TSTTB}_{R}(\mathbf{v}),
$$

where $\operatorname{TSTTB}_{R}(\mathbf{v})$ is the TSTTB at $\mathbf{v}$ given $R$. Unlike Uchida and Iida (1993), the objective function further captures the standard deviation of the TSTT to model the risk aversion of system managers. This program can be solved by existing optimization software packages.

\section{Example 3: Reliability-based system-optimal example}

The network setting in Examples 1 and 2 are used here. Assume $R$ equals 2.

By flow conservation, we have $f_{1, A C}+f_{2, A C}=1$. This together with $v_{1}=f_{1, A C}, v_{2}=f_{2, A C}$, $v_{3}=f_{1, A C}+f_{2, A C}$, (15), and (16) lead to:

$$
\begin{aligned}
E(\text { TSTT }) & =\left(1+5 v_{1}\right) v_{1}+6.07 v_{2}+\left(0.1+0.1 v_{3}\right) v_{3} \\
& =v_{1}+5\left(v_{1}\right)^{2}+6.07\left(1-v_{1}\right)+0.2, \text { and } \\
\sigma(\text { TSTT }) & =\sqrt{\left(0.07 v_{1}\right)^{2}+\left(0 v_{2}\right)^{2}+\left(0.005 v_{3}\right)^{2}+0+0+2 v_{1} v_{3}(0.00035)} \\
& =\sqrt{0.0049\left(v_{1}\right)^{2}+0.000025+2(0.00035) v_{1}} .
\end{aligned}
$$

Because $E(T S T T)+2 \sigma($ TSTT $)$ is strictly convex, the minimum flow on link 1 can be obtained by setting the derivative to zero, resulting in $v_{1}=0.49, v_{2}=0.51$, and the minimum TSTTB of 5.037."

Clearly, the solution set $\Omega_{v}$ of problem (21) is non-empty. Moreover, the set is bounded by linear constraints. It is also closed because it includes the feasible solutions on the boundary. Therefore, it is compact. Based on A2, all the terms in the TSTTB are continuous functions of link flows and hence the objective function of problem (21) is continuous with respect to link flows. According to Weierstrass' Theorem, there exists a solution to the optimization problem (21).

The solution set of problem (21) is convex because the set is formed by linear constraints. 
If the objective function of problem (21) is also strictly convex over $\Omega_{v}$, then problem (21) is a convex programming problem with a unique global minimum.

\subsection{Properties of reliability-based traffic assignment}

This section states three properties of flow patterns, which will be used to derive the upper bounds of the PoA for RUE.

Lemma 1. Given a feasible link flow pattern $\mathbf{v} \in \Omega_{v}$, the corresponding $\operatorname{TSTTB}_{R}(\mathbf{v})$ is bounded by

$$
\sum_{a \in A} t_{a} v_{a} \leq \operatorname{TSTTB}_{R}(\mathbf{v}) \leq \sum_{a \in A}\left(1+R \varepsilon_{a}\right) t_{a} v_{a}
$$

Proof. Based on Propositions 1 and 2,

$$
\operatorname{TSTTB}_{R}(\mathbf{v}) \leq \sum_{a \in A} t_{a} v_{a}+R \sqrt{\sum_{a \in A}\left(\varepsilon_{a} t_{a} v_{a}\right)^{2}+\sum_{i, j \in A, i \neq j} v_{i} v_{j}\left(\varepsilon_{i} t_{i}\right)\left(\varepsilon_{j} t_{j}\right)} .
$$

Because the condition $g_{1}^{2}+g_{2}^{2}+\ldots+g_{M}{ }^{2}+\sum_{i, j, i \neq j} g_{i} g_{j}=\left(g_{1}+g_{2}+\ldots+g_{M}\right)^{2}$ holds for any given $g_{1}, g_{2} \ldots, g_{M} \geq 0$, applying this condition to the square root term in inequality (23) gives

$$
\sqrt{\sum_{a \in A}\left(\varepsilon_{a} t_{a} v_{a}\right)^{2}+\sum_{i, j \in A, i \neq j}\left(\varepsilon_{i} t_{i} v_{i}\right)\left(\varepsilon_{j} t_{j} v_{j}\right)}=\sqrt{\left(\sum_{a \in A} \varepsilon_{a} t_{a} v_{a}\right)^{2}}=\sum_{a \in A} \varepsilon_{a} t_{a} v_{a} .
$$

Substituting (24) into (23) gives the second inequality of (22). The lower bound of $\operatorname{TSTTB}_{R}(\mathbf{v})$ in (22) can easily be obtained by setting $R=0$. This completes the proof."

Remark: There are no square root terms in the lower and upper bounds of the TSTTB in (22), which is favorable because they become linkwise additive functions. This property simplifies the derivation of a set of upper bounds of the PoA in Section 3.7.

Lemma 2. Given $\mathbf{v} \in \Omega_{v}, b_{p, r s}$ is bounded by

$$
\sum_{a \in A} t_{a} \delta_{p, r s}^{a} \leq b_{p, r s} \leq \sum_{a \in A}\left(1+\lambda \varepsilon_{a}\right) t_{a} \delta_{p, r s}^{a}, \forall p \in P_{r s}, \forall r s \in R S .
$$

Proof. The proof is similar to that for Lemma 1 except that (17) is used in the derivation instead of (20)."

Remark: Lemma 2 gives the upper and lower bounds for the path TTB and is used to prove Lemma 3.

Let $v_{a}^{R S O}$ and $v_{a}^{R U E}$ be an optimal flow on link $a \in A$ obtained by RSO and RUE assignments, respectively; $\mathbf{v}^{R S O}=\left(v_{a}^{R S O}\right)_{a \in A}$; and $\mathbf{v}^{R U E}=\left(v_{a}^{R U E}\right)_{a \in A}$. Using these notations, Lemma 3 can be stated as follows.

Lemma 3. In reliability-based traffic assignment, given $\mathbf{v}^{R S O}$ and $\mathbf{v}^{R U E}$, the following condition always holds: 


$$
\sum_{a \in A}\left[\left(1+\lambda \varepsilon_{a}\right) t_{a}\left(v_{a}^{R U E}\right) v_{a}^{R S O}-t_{a}\left(v_{a}^{R U E}\right) v_{a}^{R U E}\right] \geq 0
$$

Proof. Denote $\hat{b}_{p, r s}^{R U E}$ and $\bar{b}_{p, r s}^{R U E}$ as the upper and lower bounds of $b_{p, r s}$ in Lemma 2, i.e., $\hat{b}_{p, r s}^{R U E}=\sum_{a \in A} \delta_{p, r s}^{a}\left(1+\lambda \varepsilon_{a}\right) t_{a}\left(v_{a}^{R U E}\right)$ and $\bar{b}_{p, r s}^{R U E}=\sum_{a \in A} \delta_{p, r s}^{a} t_{a}\left(v_{a}^{R U E}\right), \forall p \in P_{r s}, \forall r s \in R S$. Then,

$$
\begin{gathered}
\hat{b}_{p, r s}^{R U E} \geq b_{p, r s}^{R U E}, \forall p \in P_{r s}, \forall r s \in R S \text {, and } \\
b_{p, r s}^{R U E} \geq \bar{b}_{p, r s}^{R U E}, \forall p \in P_{r s}, \forall r s \in R S .
\end{gathered}
$$

For a feasible flow pattern $\tilde{\mathbf{f}}=\left(\tilde{f}_{p, r s}\right)_{p \in P_{r}, r s \in R S} \in \Omega_{f}$, the path flows are non-negative. Hence, (27) can be modified to obtain

$$
\sum_{r s \in R S} \sum_{p \in P_{r s}} \tilde{f}_{p, r s} \hat{b}_{p, r s}^{R U E} \geq \sum_{r s \in R S} \sum_{p \in P_{r s}} \tilde{f}_{p, r s} b_{p, r s}^{R U E} .
$$

By subtracting $\sum_{r s \in R S} \sum_{p \in P_{r s}} f_{p, r s}^{R U E} b_{p, r s}^{R U E}$ from both sides of (29),

$$
\sum_{r s \in R S} \sum_{p \in P_{r s}} \tilde{f}_{p, r s} \hat{b}_{p, r s}^{\mathrm{RUE}}-\sum_{r s \in R S} \sum_{p \in P_{r s}} f_{p, r s}^{R U E} b_{p, r s}^{R U E} \geq \sum_{r s \in R S} \sum_{p \in P_{r s}}\left(\tilde{f}_{p, r s}-f_{p, r s}^{R U E}\right) b_{p, r s}^{R U E} .
$$

The right side of (30) is non-negative according to (19). Hence, the left side of (30) is non-negative, which implies that

$$
\sum_{r s \in R S} \sum_{p \in P_{r s}} \tilde{f}_{p, r s} \hat{b}_{p, r s}^{R U E} \geq \sum_{r s \in R S} \sum_{p \in P_{r s}} f_{p, r s}^{R U E} b_{p, r s}^{R U E} .
$$

Based on inequality (28) and $f_{p, r s}^{R U E} \geq 0$, it can be similarly deduced that

$$
\sum_{r s \in R S} \sum_{p \in P_{r s}} f_{p, r s}^{R U E} b_{p, r s}^{R U E} \geq \sum_{r s \in R S} \sum_{p \in P_{r s}} f_{p, r s}^{R U E} \bar{b}_{p, r s}^{R U E} .
$$

Based on inequalities (31) and (32),

$$
\sum_{r s \in R S} \sum_{p \in P_{r s}} \tilde{f}_{p, r s} \hat{b}_{p, r s}^{R U E} \geq \sum_{r s \in R S} \sum_{p \in P_{P s}} f_{p, r s}^{R U E} \bar{b}_{p, r s}^{R U E} .
$$

By definition, $\hat{b}_{p, r s}^{R U E}$ and $\bar{b}_{p, r s}^{R U E}$ are linkwise additive. Hence, the left and right sides of (33) can both be expressed in terms of link flows. The left side can be expressed as

$$
\begin{aligned}
\sum_{r s \in R S} \sum_{p \in P_{r s}} \tilde{f}_{p, r s} \hat{b}_{p, r s}^{R U E} & =\sum_{r s \in R S} \sum_{p \in P_{r s}} \tilde{f}_{p, r s}\left[\sum_{a \in A} \delta_{p, r s}^{a}\left(1+\lambda \varepsilon_{a}\right) t_{a}\left(v_{a}^{R U E}\right)\right] \\
& =\sum_{a \in A}\left[\sum_{r s \in R S} \sum_{p \in P_{r s}} \tilde{f}_{p, r s} \delta_{p, r s}^{a}\right]\left(1+\lambda \varepsilon_{a}\right) t_{a}\left(v_{a}^{R U E}\right) .
\end{aligned}
$$

The square-bracket term in the last equality condition of (34) equals the feasible flow on link $a$, $\tilde{v}_{a}$, associated with $\tilde{\mathbf{f}}$. Hence,

$$
\sum_{r s \in R S} \sum_{p \in P_{r s}} \tilde{f}_{p, r s} \hat{b}_{p, r s}^{R U E} \geq \sum_{a \in A} \tilde{v}_{a}\left(1+\lambda \varepsilon_{a}\right) t_{a}\left(v_{a}^{R U E}\right) .
$$

It can similarly be deduced that

$$
\sum_{r s \in R S} \sum_{p \in P_{r s}} \tilde{f}_{p, r s} \bar{b}_{p, r s}^{R U E} \geq \sum_{a \in A} v_{a}^{R U E} t_{a}\left(v_{a}^{R U E}\right) .
$$

Based on (35) and (36), the path-based inequality (33) can be expressed as a link-based inequality:

$$
\sum_{a \in A} \tilde{v}_{a}\left(1+\lambda \varepsilon_{a}\right) t_{a}\left(v_{a}^{R U E}\right) \geq \sum_{a \in A} v_{a}^{R U E} t_{a}\left(v_{a}^{R U E}\right) .
$$


Because $\tilde{v}_{a}$ is any feasible link flow, it can be replaced by $v_{a}^{R S O}$ to obtain (26) after rearranging the resulting expression. This completes the proof."

\subsection{Price of anarchy for reliability-based user equilibrium}

Similar to the PoA for UE, a PoA is proposed below to measure the relative increase in the TSTTB at RUE to that at RSO.

Definition 1. Given an RUE traffic assignment instance $(G, \mathbf{D}, \mathbf{t}, \boldsymbol{\sigma})$ and the values of $R$ and $\lambda$ representing the levels of risk aversion of the system manager and travelers, respectively, the PoA is defined as

$$
\rho_{R, \lambda}(G, \mathbf{D}, \mathbf{t}, \boldsymbol{\sigma})=\frac{\operatorname{TSTTB}_{R}\left(\mathbf{v}^{R U E}\right)}{\operatorname{TSTTB}_{R}\left(\mathbf{v}^{R S O}\right)}
$$

Definition 2. Let $I_{n, \varepsilon_{\max }}$ be the set of all instances that satisfy the following conditions:

1) The travel time functions are polynomial functions whose highest degree is not larger than a positive integer $n$, and;

2) The multiplier $\varepsilon_{a}$ of each link $a \in A$ (defined in Proposition 1) does not exceed a non-negative number $\varepsilon_{\max }$.

The PoA of $I_{n, \varepsilon_{\max }}$ is

$$
\rho_{R, \lambda}\left(I_{n, \varepsilon_{\max }}\right)=\sup _{(G, \mathbf{D}, \mathbf{t}, \boldsymbol{\sigma}) \in I_{n, \varepsilon_{\max }}} \rho_{R, \lambda}(G, \mathbf{D}, \mathbf{t}, \boldsymbol{\sigma})
$$

\section{Example 4: The PoA for reliability-based user equilibrium}

Using the information in Examples 1-3, we can determine the PoA.

In Example 2, at RUE, $f_{1, A C}=1$. Substituting it to the expression for $E(T S T T)$ and $\sigma(\mathrm{TSTT}), \quad E(\mathrm{TSTT})=6.200$ and $\sigma(\mathrm{TSTT})=0.075$. Hence, $\operatorname{TSTTB}_{R}\left(\mathbf{v}^{\mathrm{RUE}}\right)=$ $E(T S T T)+2 \sigma(T S T T)=6.350$. In Example 3, the TSTTB at RSO is 5.037. Thus, $\rho_{R, \lambda}=6.350 / 5.037=1.261$."

The non-uniqueness of RSO and RUE solutions does not affect the PoA because all multiple RSO solutions of the same instance give the same TSTTB and all multiple RUE solutions of the same instance give the same TSTTB.

\subsection{Derivation of a set of upper bounds of the price of anarchy}

Consider an RUE traffic assignment instance $(G, \mathbf{D}, \mathbf{t}, \boldsymbol{\sigma}) \in I_{n, \varepsilon_{\max }}$, whose RUE and RSO link flows are $\mathbf{v}^{R U E}$ and $\mathbf{v}^{R S O}$, respectively. Let $t_{a}^{R U E}=t_{a}\left(v_{a}^{R U E}\right)$ and $t_{a}^{R S O}=t_{a}\left(v_{a}^{R S O}\right)$, $\forall a \in A$. In the following, we derive a set of upper bounds of the PoA for this instance, given the parameter values of the risk aversion of system manager and travelers. i.e., a set of upper bounds of $\rho_{R, \lambda}(G, \mathbf{D}, \mathbf{t}, \boldsymbol{\sigma})$. To simplify the notation, the notation $\rho_{R, \lambda}$ is used to represent 
$\rho_{R, \lambda}(G, \mathbf{D}, \mathbf{t}, \boldsymbol{\sigma})$ in this section.

The numerator and denominator in $\rho_{R, \lambda}(G, \mathbf{D}, \mathbf{t}, \boldsymbol{\sigma})$ (see (38)) are both not link-separable. The PoA is enlarged so that both they become link-separable and some of the techniques used to derive the upper bound of the PoA of UE can be applied to that for RUE.

Define

$$
\Omega_{\Phi}=\Omega_{\Phi^{\prime}} \cap \Omega_{\Phi^{\prime \prime}},
$$

where

$$
\begin{gathered}
\Omega_{\Phi^{\prime}}=\left\{k^{\prime} \mid 0<k^{\prime} \leq \frac{1}{1+\lambda \varepsilon_{\max }}\right\}, \\
\Omega_{\Phi^{\prime \prime}}=\left\{k^{\prime \prime} \mid 0<k^{\prime \prime} \leq\left(\frac{1}{n+\Delta}\right)^{n}\left(\frac{n+1}{1+\lambda \varepsilon_{\max }}\right)^{n+1}\right\},
\end{gathered}
$$

and $\mathrm{D}$ is a small positive number less than one. The above conditions lead to

$$
\Omega_{\Phi}=\left\{k \mid 0<k \leq \min \left(\frac{1}{1+\lambda \varepsilon_{\max }},\left(\frac{1}{n+\Delta}\right)^{n}\left(\frac{n+1}{1+\lambda \varepsilon_{\max }}\right)^{n+1}\right)\right\} .
$$

Remark 1: Both $\Omega_{\Phi^{\prime}}$ and $\Omega_{\Phi^{\prime \prime}}$ are non-empty sets with the common lower bound of zero. Thus, $\Omega_{\Phi}$ is non-empty.

Remark 2: $\Omega_{\Phi}$ depends on the parameter values $n, \lambda$, and $\varepsilon_{\max }$ and the small positive number $\mathrm{D}$.

Lemma 4. Given a non-negative link flow $r_{a}$ (e.g., an RUE link flow), $a \in A$, for any feasible flow on that link, i.e., $x_{a} \geq 0$, the following is true:

$$
k_{a m} x_{a}{ }^{m} x_{a}-\Phi\left\{\left(1+\lambda \varepsilon_{a}\right) k_{a m} r_{a}^{m} x_{a}-k_{a m} r_{a}{ }^{m} r_{a}\right\} \geq \theta\left(\Phi, m, \lambda, \varepsilon_{a}\right) k_{a m} r_{a}{ }^{m} r_{a}, \forall a \in A,
$$

where $m \hat{\imath} \Phi_{+}$is a power to $x_{a} ; \varepsilon_{a}$ equals $\sigma\left(T_{a}\right) / k_{a 0} ; k_{a m}$ is a positive coefficient associated with $x_{a}{ }^{m}, \Phi \in \Omega_{\Phi}$, and $\theta\left(\Phi, m, \lambda, \varepsilon_{a}\right)$ is defined as

$$
\theta\left(\Phi, m, \lambda, \varepsilon_{a}\right)=\Phi-m\left(\frac{\Phi\left(1+\lambda \varepsilon_{a}\right)}{m+1}\right)^{\frac{m+1}{m}} \text {. }
$$

Proof. See Appendix B.

Remark 1: Lemma 4 can also be evoked by any non-negative numbers $r_{a}$ and $x_{a}$.

Remark 2: The power $m$ cannot be zero because $\theta\left(\Phi, m, \lambda, \varepsilon_{a}\right)$ is not defined at $m=0$. However, $m=0$ is still considered in the derivation of the PoA. See the proof of Lemma 6 in Appendix D.

Remark 3: Lemma 4 still holds if $\Phi$ is positive. 
Lemma 5. The function $\theta\left(\Phi, m, \lambda, \varepsilon_{a}\right)$ defined in (45) is strictly decreasing in terms of $m \hat{\imath} \Phi_{+}$and positive for any fixed value of $\Phi$ taking from the set $\Omega_{\Phi}$ defined by Eq. (43) (i.e., for any $\Phi \in \Omega_{\Phi}$ ), where $\varepsilon_{a}$ equals $\sigma\left(T_{a}\right) / k_{a 0}$ and $m \hat{\imath} \Phi_{+}$.

Proof. See Appendix C.

Lemma 6. For each individual link $a \in A$, the following is true:

$$
t_{a}^{R S O} v_{a}^{R S O}-\Phi\left\{\left(1+\lambda \varepsilon_{a}\right) t_{a}^{R U E} v_{a}^{R S O}-t_{a}^{R U E} v_{a}^{R U E}\right\} \geq \theta\left(\Phi, n, \lambda, \varepsilon_{a}\right) t_{a}^{R U E} v_{a}^{R U E}, \forall a \in A
$$

and for any fixed value of $\Phi$ taking from the set $\Omega_{\Phi}$, in which $n \hat{\imath} \Phi_{+}$is the largest degree in all of the polynomial link performance functions, $\varepsilon_{a}$ equals $\sigma\left(T_{a}\right) / k_{a 0}$, and $\theta\left(\Phi, n, \lambda, \varepsilon_{a}\right)$ follows the definition in (45).

Proof. See Appendix D.

Proposition 4. For each individual link $a \in A$, the following is true:

$$
\frac{\left(1+R \varepsilon_{a}\right) t_{a}^{R U E} v_{a}^{R U E}}{t_{a}^{R S O} v_{a}^{R S O}-\Phi\left\{\left(1+\lambda \varepsilon_{a}\right) t_{a}^{R U E} v_{a}^{R S O}-t_{a}^{R U E} v_{a}^{R U E}\right\}} \leq \frac{1+R \varepsilon_{a}}{\theta\left(\Phi, n, \lambda, \varepsilon_{a}\right)}, \forall a \in A
$$

for any $\Phi \in \Omega_{\Phi}$, where $n \hat{\imath} \Phi_{+} ; \varepsilon_{a}=\sigma\left(T_{a}\right) / k_{a 0}$, and $\theta\left(\Phi, n, \lambda, \varepsilon_{a}\right)$ follows the definition in (45).

Proof. Because $\Phi \in \Omega_{\Phi}$, according to Lemma 5, $\theta\left(\Phi, n, \lambda, \varepsilon_{a}\right)$ is positive. Hence, both the left and right sides of (46) in Lemma 6 are non-negative. If $v_{a}^{R U E}>0$, both sides of (46) are positive. Dividing $\left(1+R \varepsilon_{a}\right) t_{a}^{R U E} v_{a}^{R U E}$ by the left and right sides of (46) respectively, we have

$$
\begin{aligned}
\frac{\left(1+R \varepsilon_{a}\right) t_{a}^{R U E} v_{a}^{R U E}}{t_{a}^{R S O} v_{a}^{R S O}-\Phi\left\{\left(1+\lambda \varepsilon_{a}\right) t_{a}^{R U E} v_{a}^{R S O}-t_{a}^{R U E} v_{a}^{R U E}\right\}} & \leq \frac{\left(1+R \varepsilon_{a}\right) t_{a}^{R U E} v_{a}^{R U E}}{\theta\left(\Phi, n, \lambda, \varepsilon_{a}\right) t_{a}^{R U E} v_{a}^{R U E}} \\
& \leq \frac{1+R \varepsilon_{a}}{\theta\left(\Phi, n, \lambda, \varepsilon_{a}\right)} .
\end{aligned}
$$

When $v_{a}^{R U E}=0$, there are two cases. If $v_{a}^{R S O}=0$, the denominator of the left side of (47) equals zero. However, this case is exclusive from our consideration because the link is exclusive from the network if it is unused in all circumstances. If $v_{a}^{R S O}>0$, the denominator is always larger than zero because $\Phi\left(1+\lambda \varepsilon_{a}\right) \leq 1$ and

$$
\begin{aligned}
t_{a}^{R S O} v_{a}^{R S O}-\Phi\left\{\left(1+\lambda \varepsilon_{a}\right) t_{a}^{R U E}(0) v_{a}^{R S O}-t_{a}^{R U E}(0) \cdot 0\right\} & \geq\left(t_{a}^{R S O}-\Phi\left(1+\lambda \varepsilon_{a}\right) t_{a}^{0}\right) v_{a}^{R S O} \\
& >0 .
\end{aligned}
$$

However, the left side of (47) still equals zero (because the numerator is zero), which is obviously not greater than the right side of (47). This completes the proof."

Proposition 5. Define $\hat{\rho}_{R, \lambda}(\Phi)$ as follows: 


$$
\hat{\rho}_{R, \lambda}(\Phi)=\frac{\sum_{a \in A}\left(1+R \varepsilon_{a}\right) t_{a}^{R U E} v_{a}^{R U E}}{\sum_{a \in A} t_{a}^{R S O} v_{a}^{R S O}-\Phi\left\{\sum_{a \in A}\left(\left(1+\lambda \varepsilon_{a}\right) t_{a}^{R U E} v_{a}^{R S O}-t_{a}^{R U E} v_{a}^{R U E}\right)\right\}},
$$

where $\Phi \in \Omega_{\Phi}$. Then,

$$
\rho_{R, \lambda} \leq \hat{\rho}_{R, \lambda}(\Phi)
$$

Proof. Substituting $\mathbf{v}^{R U E}$ and $\mathbf{v}^{R S O}$ into the equalities in Lemma 1, respectively, we obtain

$$
\begin{gathered}
\operatorname{TSTTB}_{R}\left(\mathbf{v}^{R U E}\right) \leq \sum_{a \in A}\left(1+R \varepsilon_{a}\right) t_{a}^{R U E} v_{a}^{R U E}, \text { and } \\
\sum_{a \in A} t_{a}^{R S O} v_{a}^{R S O} \leq \operatorname{TSTTB}_{R}\left(\mathbf{v}^{R S O}\right) .
\end{gathered}
$$

Both the left and right sides of (51) and (52) are positive due to A2. Therefore, both sides of (51) can be divided by $\sum_{a \in A} t_{a}^{R S O} v_{a}^{R S O}$, to obtain

$$
\frac{\operatorname{TSTTB}_{R}\left(\mathbf{v}^{R U E}\right)}{\sum_{a \in A} t_{a}\left(v_{a}^{R S O}\right) v_{a}^{R S O}} \leq \frac{\sum_{a \in A}\left(1+R \varepsilon_{a}\right) t_{a}\left(v_{a}^{R U E}\right) v_{a}^{R U E}}{\sum_{a \in A} t_{a}\left(v_{a}^{R S O}\right) v_{a}^{R S O}} .
$$

According to condition (52), the left side of (53) is bounded by $\rho_{R, \lambda}$, leading to

$$
\rho_{R, \lambda} \leq \frac{\sum_{a \in A}\left(1+R \varepsilon_{a}\right) t_{a}\left(v_{a}^{R U E}\right) v_{a}^{R U E}}{\sum_{a \in A} t_{a}\left(v_{a}^{R S O}\right) v_{a}^{R S O}} .
$$

Because $\left\{\sum_{a \in A}\left(\left(1+\lambda \varepsilon_{a}\right) t_{a}^{R U E} v_{a}^{R S O}-t_{a}^{R U E} v_{a}^{R U E}\right)\right\}$ is non-negative (see Lemma 3) and $\Phi$ is positive, the denominator on the right side of (54) has this property:

$$
\sum_{a \in A} t_{a}^{R S O} v_{a}^{R S O} \geq \sum_{a \in A} t_{a}^{R S O} v_{a}^{R S O}-\Phi\left\{\sum_{a \in A}\left(\left(1+\lambda \varepsilon_{a}\right) t_{a}^{R U E} v_{a}^{R S O}-t_{a}^{R U E} v_{a}^{R U E}\right)\right\} .
$$

Furthermore, based on Lemmas 5 and 6 and the non-negativity of link flow and travel time, we have

$$
t_{a}^{R S O} v_{a}^{R S O}-\Phi\left\{\left(1+\lambda \varepsilon_{a}\right) t_{a}^{R U E} v_{a}^{R S O}-t_{a}^{R U E} v_{a}^{R U E}\right\} \geq \theta\left(\Phi, n, \lambda, \varepsilon_{a}\right) t_{a}^{R U E} v_{a}^{R U E} \geq 0, \quad \forall a \in A,
$$

where the second inequality becomes strict when $v_{a}^{R U E}>0$ holds and becomes equality when $v_{a}^{R U E}=0$ holds. Because demand is positive by definition, not all RUE link flows ( $\left.v_{a}^{R U E}\right)$ are zero. Hence, taking summation on both sides of (56) over all links, we get

$$
\sum_{a \in A} t_{a}^{R S O} v_{a}^{R S O}-\Phi\left\{\sum_{a \in A}\left(\left(1+\lambda \varepsilon_{a}\right) t_{a}^{R U E} v_{a}^{R S O}-t_{a}^{R U E} v_{a}^{R U E}\right)\right\}>0 .
$$

Dividing the numerator on the right side of (54) by both the left and right sides of (55) respectively, we have 


$$
\frac{\sum_{a \in A}\left(1+R \varepsilon_{a}\right) t_{a}\left(v_{a}^{R U E}\right) v_{a}^{R U E}}{\sum_{a \in A} t_{a}\left(v_{a}^{R S O}\right) v_{a}^{R S O}} \leq \hat{\rho}_{R, \lambda}(\Phi) .
$$

Based on (54) and (58), we obtain (50). This completes the proof. "

Proposition 6. The following is true:

$$
\hat{\rho}_{R, \lambda}(\Phi) \leq \frac{1+R \varepsilon_{\max }}{\theta\left(\Phi, n, \lambda, \varepsilon_{\max }\right)}
$$

for any $\Phi \in \Omega_{\Phi}$, where $\theta\left(\Phi, n, \lambda, \varepsilon_{\max }\right)$ follows the definition in (45).

Proof. Based on the proof of Proposition 4, if $\Phi \in \Omega_{\Phi}$, the denominator of the left side of (47) is positive. Because $\frac{\sum_{m^{\prime \prime}=1}^{M} p_{m^{\prime \prime}}}{\sum_{m^{\prime \prime}=1}^{M} q_{m^{\prime \prime}}} \leq \max _{m^{\prime \prime}=1,2 \ldots M}\left(\frac{p_{m^{\prime \prime}}}{q_{m^{\prime \prime}}}\right)$ for any given $p_{m^{\prime \prime}} \geq 0, \quad q_{m^{\prime \prime}}>0$, $m^{\prime \prime}=1,2 \ldots, M, M i ̂ \Phi_{+}$, apply this condition to (47) to obtain

$$
\frac{\sum_{a \in A}\left(1+R \varepsilon_{a}\right) t_{a}^{R U E} v_{a}^{R U E}}{\sum_{a \in A} t_{a}^{R S O} v_{a}^{R S O}-\Phi \sum_{a \in A}\left\{\left(1+\lambda \varepsilon_{a}\right) t_{a}^{R U E} v_{a}^{R S O}-t_{a}^{R U E} v_{a}^{R U E}\right\}} \leq \max _{a \in A} \frac{1+R \varepsilon_{a}}{\theta\left(\Phi, n, \lambda, \varepsilon_{a}\right)} .
$$

The value of $\left(1+R \varepsilon_{a}\right)$ is non-decreasing with an increase in the value of $\varepsilon_{a}$ because $R$ is non-negative. Moreover, given $\Phi \in \Omega_{\Phi}, n$, and $\lambda$, the value of $\theta\left(\Phi, n, \lambda, \varepsilon_{a}\right)$ is decreasing with an increase in the value of $\varepsilon_{a}$ (for $0 \leq \varepsilon_{a} \leq \varepsilon_{\max }$ ) because $\theta\left(\Phi, n, \lambda, \varepsilon_{a}\right)=\Phi-n\left(\frac{\Phi\left(1+\lambda \varepsilon_{a}\right)}{n+1}\right)^{\frac{n+1}{n}}$ and the second term must be negative and the power of $\left(1+\lambda \varepsilon_{a}\right)$ in the second term, i.e., $\frac{n+1}{n}$, must be larger than one. Thus,

$$
\max _{a \in A} \frac{1+R \varepsilon_{a}}{\theta\left(\Phi, n, \lambda, \varepsilon_{a}\right)} \leq \frac{1+R \varepsilon_{\max }}{\theta\left(\Phi, n, \lambda, \varepsilon_{\max }\right)} .
$$

From conditions (60) and (61), the same statement in Proposition 6 can be concluded. This completes the proof."

Theorem 1. The PoA of an RUE traffic assignment instance $(G, \mathbf{D}, \mathbf{t}, \sigma)$ that belongs to the set $I_{n, \varepsilon_{\max }}$ is bounded above as follows:

$$
\rho_{R, \lambda}(G, \mathbf{D}, \mathbf{t}, \boldsymbol{\sigma}) \leq \frac{1+R \varepsilon_{\max }}{\theta\left(\Phi, n, \lambda, \varepsilon_{\max }\right)}, \forall(G, \mathbf{D}, \mathbf{t}, \boldsymbol{\sigma}) \in I_{n, \varepsilon_{\max }},
$$

where

$$
\Phi \in \Omega_{\Phi}=\left\{k \mid 0<k \leq \min \left(\frac{1}{1+\lambda \cdot \varepsilon_{\max }},\left(\frac{1}{n+\Delta}\right)^{n}\left(\frac{n+1}{1+\lambda \varepsilon_{\max }}\right)^{n+1}\right)\right\},
$$




$$
\theta\left(\Phi, n, \lambda, \varepsilon_{\max }\right)=\Phi-n\left[\Phi\left(1+\lambda \varepsilon_{\max }\right)(n+1)^{-1}\right]^{(n+1) / n} \text {, and }
$$

$\Delta$ is a small positive number less than one.

Proof. It follows directly from Propositions 5 and 6 that are true for any given instance $(G, \mathbf{D}, \mathbf{t}, \boldsymbol{\sigma}) \in I_{n, \varepsilon_{\max }}$.

Remark 1: The results in Theorem 1 implies that $\rho_{R, \lambda}\left(I_{n, \varepsilon_{\max }}\right)$ is also bounded by the right side of (62).

Remark 2: Note that the right side of (62) indeed defines a set of upper bounds of the PoA because $\Phi$ can take any values within $\Omega_{\Phi}$. In practice, the minimum upper bound is the most preferable. Denote it as $\rho_{R, \lambda}^{\min }(G, \mathbf{D}, \mathbf{t}, \boldsymbol{\sigma})$, or simply $\rho_{R, \lambda}^{\min }$, which is attained at a particular value $\Phi^{*} \in \Omega_{\Phi}$ as discussed below.

Theorem 2. The minimum upper bound $\rho_{R, \lambda}^{\min }$ occurs at

$$
\Phi^{*}=\min \left[\Phi^{s}, \Phi^{\prime}\right]
$$

where

$$
\begin{gathered}
\Phi^{s}=(n+1)\left(\frac{1}{1+\lambda \varepsilon_{\max }}\right)^{n+1} \text {, and } \\
\Phi^{\prime}=\frac{1}{1+\lambda \varepsilon_{\max }} .
\end{gathered}
$$

Moreover,

$$
\rho_{R, \lambda}^{\min }=\left\{\begin{array}{cc}
\left(1+R \varepsilon_{\max }\right)\left(\frac{1}{\left(1+\lambda \varepsilon_{\max }\right)}-n\left(\frac{1}{n+1}\right)^{\frac{n+1}{n}}\right)^{-1} & \text { if } \Phi^{*}=\Phi^{\prime}<\Phi^{s} ; \\
\left(1+R \varepsilon_{\max }\right)\left(1+\lambda \varepsilon_{\max }\right)^{n+1} & \text { if } \Phi^{*}=\Phi^{s} \leq \Phi^{\prime} .
\end{array}\right.
$$

Proof. The factor $\Phi$ only appears in the denominator on the right side of (62), i.e., $\theta\left(\Phi, n, \lambda, \varepsilon_{\max }\right)$, and the maximum denominator value gives $\rho_{R, \lambda}^{\min }$. Hence, the proof can be reduced to determine a global maximum $\Phi^{*}$ of $\theta\left(\Phi, n, \lambda, \varepsilon_{\max }\right)$.

The first and second derivatives of $\theta\left(\Phi, n, \lambda, \varepsilon_{\max }\right)$ in terms of $\Phi$ are

$$
\begin{aligned}
& \frac{d \theta\left(\Phi, n, \lambda, \varepsilon_{\max }\right)}{d \Phi}=1-(n+1)\left(\frac{1+\lambda \varepsilon_{\max }}{n+1}\right)^{\frac{n+1}{n}} \Phi^{\frac{1}{n}} \text { and } \\
& \frac{d^{2} \theta\left(\Phi, n, \lambda, \varepsilon_{\max }\right)}{d \Phi^{2}}=-\frac{1}{n}\left(\frac{1}{n+1}\right)^{\frac{1}{n}}\left(1+\lambda \varepsilon_{\max }\right)^{\frac{n+1}{n}} \Phi^{\frac{1-n}{n}},
\end{aligned}
$$


respectively. The second derivative (70) is always negative because $n$ is positive and the other inputs are non-negative. This implies that $\theta\left(\Phi, n, \lambda, \varepsilon_{\max }\right)$ is a strictly concave function of $\Phi$, and a global maximum can only occur at either

1) the stationary point $\Phi^{s}=(n+1)\left(\frac{1}{1+\lambda \varepsilon_{\max }}\right)^{n+1}$, where the first derivative (69) equals zero, or

2) a boundary of the feasible range of $\Phi$.

The first case occurs when the feasible range contains $\Phi^{s}$ because the boundaries are always not binding at optimality. Otherwise, the second case occurs. Moreover, $\theta\left(\Phi, n, \lambda, \varepsilon_{\max }\right)$ is a strictly increasing function of $\Phi$ when $\Phi<\Phi^{s}$. Therefore, the global maximum occurs at the upper boundary of the feasible range for the second case when $\Phi<\Phi^{s}$.

Let $\Phi^{\prime \prime}$ be $\left(\frac{1}{n+\Delta}\right)^{n}\left(\frac{n+1}{1+\lambda \varepsilon_{\max }}\right)^{n+1}$. Consider two cases: $\Phi^{\prime}<\Phi^{\prime \prime}$ and $\Phi^{\prime} \geq \Phi^{\prime \prime}$.

First, when $\Phi^{\prime}<\Phi^{\prime \prime}$, the feasible range becomes $0<\Phi \leq \Phi^{\prime}$ according to (43). There are two scenarios in this case, i.e., $\Phi^{s} \leq \Phi^{\prime}$ and $\Phi^{\prime}<\Phi^{s}$. In the former, $\Phi^{s}$ exists within the feasible range of $\Phi$ and hence $\Phi^{*}=\Phi^{s} \leq \Phi^{\prime}$. In the latter, $\theta\left(\Phi, n, \lambda, \varepsilon_{\max }\right)$ is a strictly increasing function in the feasible range considered whose maximum is reached at the upper boundary $\Phi^{\prime}$. Therefore, $\Phi^{*}=\Phi^{\prime}<\Phi^{s}$. In summary, $\Phi^{*}=\min \left(\Phi^{\prime}, \Phi^{s}\right)$.

Second, when $\Phi^{\prime} \geq \Phi^{\prime \prime}$, the feasible range becomes $0<\Phi \leq \Phi^{\prime \prime}$ according to (43). The upper boundary $\Phi^{\prime \prime}$ must be greater than $\Phi^{s}$ because for any positive integer of $n$,

$$
\begin{aligned}
& \Phi^{s}=(n+1)\left(\frac{1}{1+\lambda \varepsilon_{\max }}\right)^{n+1} \\
& <\left(\frac{n+1}{n+\Delta}\right)^{n}(n+1)\left(\frac{1}{1+\lambda \varepsilon_{\max }}\right)^{n+1}=\Phi^{\prime \prime} .
\end{aligned}
$$

This means that $\Phi^{s}$ must exist within the feasible range of $\Phi$. Thus, $\Phi^{*}=\Phi^{s}<\Phi^{\prime \prime} \leq \Phi^{\prime}$.

Summarizing two cases, $\Phi^{*}=\min \left(\Phi^{\prime}, \Phi^{s}\right)$, which is identical to (65).

Substituting (65)-(67) to the right side of the inequality in Theorem 1, we can obtain $\rho_{R, \lambda}^{\min }$ as shown in (68). This completes the proof."

\section{Example 5. Minimum upper bound of the PoA}

Using the results in Theorem 2, we can determine $\rho_{R, \lambda}^{\min }$ for the instance described in Examples 1-4.

The RUE traffic assignment instance in Example 1 belongs to the set $I_{n, \varepsilon_{\max }}$, in which $n=1$ and $\varepsilon_{\max }=0.07$.

Based on (66) and (67), $\Phi^{s}=(n+1)\left(1+\lambda \varepsilon_{\max }\right)^{-n-1}=1.7469 \quad$ and 
$\Phi^{\prime}=\left(1+\lambda \varepsilon_{\max }\right)^{-1}=0.9346$. Thus, $\Phi^{s}>\Phi^{\prime}$. As a result,

$\rho_{R, \lambda}^{\min }=\left(1+R \varepsilon_{\max }\right)\left(\frac{1}{\left(1+\lambda \varepsilon_{\max }\right)}-n\left(\frac{1}{n+1}\right)^{\frac{n+1}{n}}\right)^{-1}=1.665$ (see (68)). .

Remark: The PoA in Example 4 is 1.261, which is bounded by $\rho_{R, \lambda}^{\min }$. It does not equal $\rho_{R, \lambda}^{\min }$ because the PoA for the instance considered is not the worst case, and there is a loss in the tightness in deriving $\rho_{R, \lambda}^{\min }$.

Theorem 3. The set of upper bounds of the PoA for reliability-based traffic assignment is independent of the network topology $G$ and demand $\mathbf{D}$.

Proof. The right side of (62) does not depend on $G$ and D. Thus, the statement follows.

\subsection{Analyses of the minimum upper bound of the price of anarchy}

This section examines how the value of the minimum bound is independently affected by its inputs: $R, n, \lambda$, and $\varepsilon_{\max }$. For the purpose of analysis, $\rho_{R, \lambda}^{\min }$ is rewritten as follows:

in which

$$
\rho_{R, \lambda}^{\min }=\left\{\begin{array}{l}
\left(1+R \varepsilon_{\max }\right) \theta\left(\Phi^{\prime}, n, \lambda, \varepsilon_{\max }\right)^{-1} \text { for } \Phi^{\prime}<\Phi^{s} \\
\left(1+R \varepsilon_{\max }\right) \theta\left(\Phi^{s}, n, \lambda, \varepsilon_{\max }\right)^{-1} \text { for } \Phi^{s} \leq \Phi^{\prime}
\end{array}\right.
$$

$$
\begin{gathered}
\theta\left(\Phi^{\prime}, n, \lambda, \varepsilon_{\max }\right)=\frac{1}{1+\lambda \varepsilon_{\max }}-n\left(\frac{1}{n+1}\right)^{\frac{n+1}{n}} \text {, and } \\
\theta\left(\Phi^{s}, n, \lambda, \varepsilon_{\max }\right)=\left(\frac{1}{1+\lambda \varepsilon_{\max }}\right)^{n+1} .
\end{gathered}
$$

Proposition 8. The minimum upper bound $\rho_{R, \lambda}^{\min }$ is linearly increasing in terms of $R$.

Proof. According to (72), the denominator is independent of $R$ in each of the two cases. Moreover, the optimal value of $\Phi^{*}$ is also independent of $R$. Therefore, when examining the relation between $\rho_{R, \lambda}^{\min }$ and $R$ in each case, the denominator can be treated as a constant, which is positive according to Lemma 5 under $\Phi^{*} \in \Omega_{\Phi}$. Moreover, in each case, the factor $R$ is only involved in the numerator of $\rho_{R, \lambda}^{\min }$. Its coefficient is positive, and the power is one. Thus, $\rho_{R, \lambda}^{\min }$ is linearly increasing in terms of $R$ when other parameter values are fixed. Furthermore, when they are fixed, only one of the two cases occurs. As such, the statement follows. This completes the proof."

Proposition 9. The minimum upper bound $\rho_{R, \lambda}^{\min }$ is strictly increasing in terms of $n$. 
Proof. The parameter $n$ affects the value of $\Phi^{*}$, and both $n$ and $\Phi^{*}$ are only involved in the denominator of (72). Hence, the properties of the denominator can be made a focus. Two mutually exclusive cases are considered.

First, when $(n+1)^{\frac{1}{n}}>1+\lambda \varepsilon_{\max }$ for $n \hat{\imath} \Phi_{+}, \quad \Phi^{\prime}<\Phi^{s}$, and according to (72), the denominator is $\theta\left(\Phi^{\prime}, n, \lambda, \varepsilon_{\max }\right)$ shown in (73). The first derivative of $\theta\left(\Phi^{\prime}, n, \lambda, \varepsilon_{\max }\right)$ in terms of $n$ is

$$
\frac{d}{d n} \theta\left(\Phi^{\prime}, n, \lambda, \varepsilon_{\max }\right)=\frac{1}{n}\left(\frac{1}{n+1}\right)^{\frac{n+1}{n}} \ln \left(\frac{1}{n+1}\right) .
$$

Because $\frac{1}{n+1}$ is smaller than 1 , the first derivative (75) is always negative. Thus, $\theta\left(\Phi^{\prime}, n, \lambda, \varepsilon_{\max }\right)$ must be a strictly decreasing function of $n$.

Second, when $(n+1)^{\frac{1}{n}} \leq 1+\lambda \varepsilon_{\max }$ for $n \hat{\imath} \Phi_{+}, \Phi^{s} \leq \Phi^{\prime}$, and the denominator equals $\theta\left(\Phi^{s}, n, \lambda, \varepsilon_{\max }\right)$ shown in (74). Because $\frac{1}{1+\lambda \varepsilon_{\max }}$ is smaller than one, the function $\theta\left(\Phi^{s}, n, \lambda, \varepsilon_{\max }\right)$ must be a strictly decreasing function of $n$.

In summary, the denominator must be a strictly decreasing function of $n$, and hence $\rho_{R, \lambda}^{\min }$ must be strictly increasing in terms of $n$. This completes the proof.

Remark: The minimum upper bound is only an estimate to the PoA. It cannot be concluded whether the PoA (strictly) increases with the maximum degree. This is left for future research.

Proposition 10. The minimum upper bound $\rho_{R, \lambda}^{\min }$ is strictly increasing in terms of $\lambda$.

Proof. The proof is similar to that for Proposition 9. When $0 \leq \lambda<\left[(n+1)^{\frac{1}{n}}-1\right] / \varepsilon_{\max }$, $\Phi^{\prime}<\Phi^{s}$, and the denominator equals $\theta\left(\Phi^{\prime}, n, \lambda, \varepsilon_{\max }\right)$, which is clearly a strictly decreasing function of $\lambda$. However, when $\lambda \geq\left[(n+1)^{\frac{1}{n}}-1\right] / \varepsilon_{\max }$, the denominator equals $\theta\left(\Phi^{s}, n, \lambda, \varepsilon_{\max }\right)$, which is also a decreasing function of $\lambda$. Hence, the statement follows. This completes the proof."

Remark 1: When $\Phi^{s} \leq \Phi^{\prime}$, the second derivative of $\rho_{R, \lambda}^{\min }$ in terms of $\lambda$ is $(n+1) n\left(1+R \varepsilon_{\max }\right)\left(1+\lambda \varepsilon_{\max }\right)^{n-1}$. Because the second derivative must be positive, $\rho_{R, \lambda}^{\min }$ is a convex function of $\lambda$ when $\Phi^{s} \leq \Phi^{\prime}$. However, when $\Phi^{\prime}<\Phi^{s}$, the second derivative is $\frac{\left(1+R \varepsilon_{\max }\right)\left\{\left(1+\lambda \varepsilon_{\max }\right)^{-1} \theta\left(\Phi^{\prime}, n, \lambda, \varepsilon_{\max }\right)^{-1}-2\right\}}{\left(1+\lambda \varepsilon_{\max }\right) \theta\left(\Phi^{\prime}, n, \lambda, \varepsilon_{\max }\right)^{2}}$, which may not always be positive. Hence, it cannot be concluded that $\rho_{R, \lambda}^{\min }$ is a convex function of $\lambda$ when $\Phi^{\prime}<\Phi^{s}$. 
Remark 2: According to Propositions 8 and 10, the minimum upper bound of the PoA increases either with an increasing level of risk aversion of the system manager or of the travelers. However, the effects of their risk aversions on the bound may be different because it may increase nonlinearly with $\lambda$ but increases linearly with $R$.

Proposition 11. The minimum upper bound $\rho_{R, \lambda}^{\min }$ is strictly increasing in terms of $\varepsilon_{\max }$.

Proof. The numerator in (72) in both cases increases with $\varepsilon_{\max }$. Following the proof for Proposition 10, it can be concluded that the denominator is strictly decreasing in terms of $\varepsilon_{\max }$. Hence, the statement follows. This completes the proof."

Remark: The convexity of $\rho_{R, \lambda}^{\min }$ in terms of $\varepsilon_{\max }$ can similarly not be concluded.

Similar to Yang et al. (2008), $\rho_{R, \lambda}^{\text {min }}$ includes the upper bound of Roughgarden (2005) as a special case as stated below:

Corollary 1. If either 1) $\varepsilon_{\max }=0$ or 2) $R=\lambda=0$ holds, then $\rho_{R, \lambda}^{\min }$ is reduced to $\rho^{\prime}$, the upper bound obtained by Roughgarden (2005).

Proof. If either sufficient condition 1) or 2) holds, $\Phi^{s}=n+1$, and $\Phi^{\prime}=1<\Phi^{s}$. Substituting $\varepsilon_{\max }=0$ or $R=\lambda=0$ into the upper equation in (73), $\rho_{R, \lambda}^{\min }=\rho^{\prime}$. This completes the proof.

When $\varepsilon_{\max }=0$, there is no network uncertainty. When $R=\lambda=0$, both the users and system manager are risk neutral and do not consider safety margins. In both cases, the TSTTB and RUE traffic assignment are reduced to the TSTT and UE traffic assignment, respectively. Hence, it is reasonable that $\rho_{R, \lambda}^{\min }$ is reduced to the upper bound of Roughgarden (2005) if either sufficient condition is satisfied.

\section{Example 6: The effects of $R, \lambda$, and $\varepsilon_{\max }$}

To illustrate the effects of $R, \lambda$, and the standard deviation of link travel time (or $\varepsilon_{\max }$ ) on the minimum upper bound, Figures 2a, b, and c are plotted based on the settings in Examples 1-3 that except the standard deviation $\sigma\left(T_{1}\right)$ is 0.01 . In each of these figures, only one of the three values is varied, and the minimum upper bound and the corresponding lower bound of the PoA of the instance in interest are also shown. 


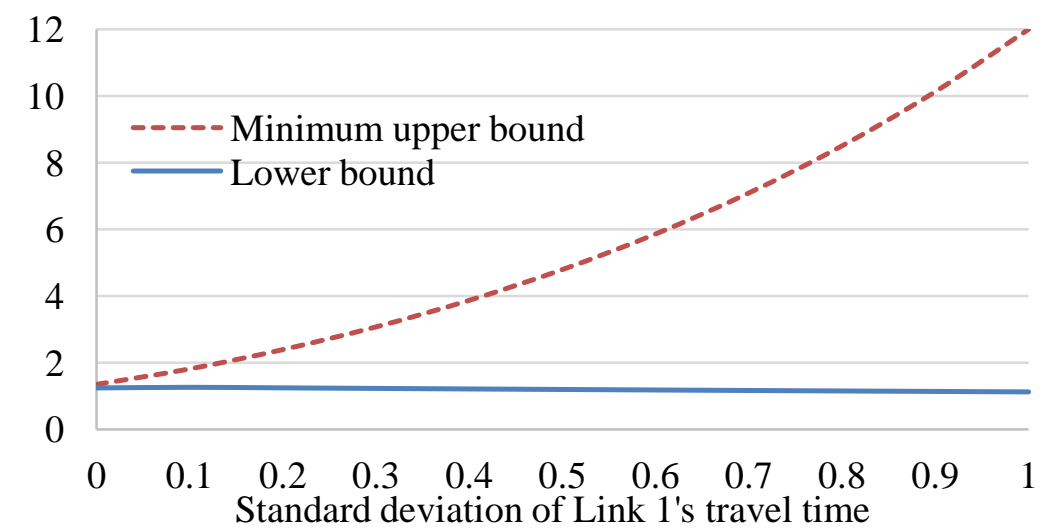

(a) the effect of standard deviation

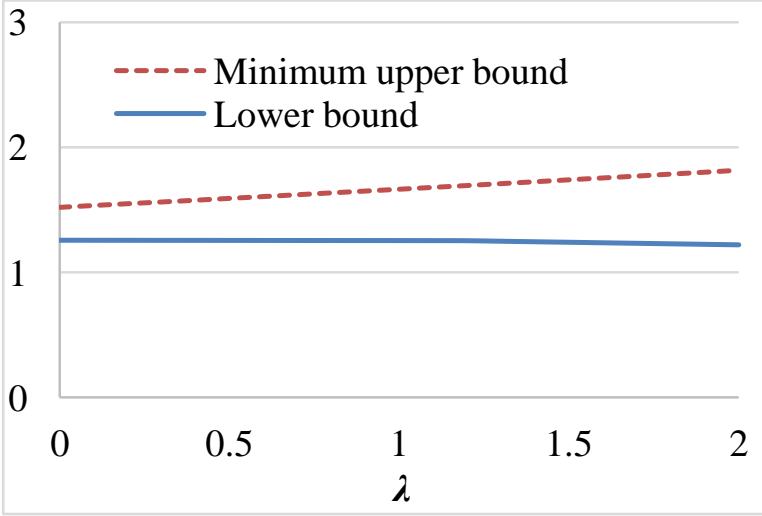

(b) the effect of $\lambda$

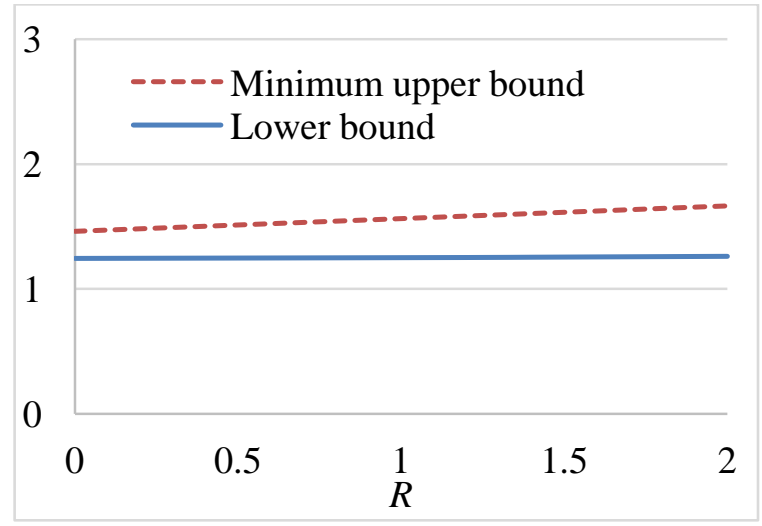

(c) the effect of $R$

Figure 2 Lower bound and minimum upper bounds subject to the variation of $\sigma\left(T_{1}\right), \lambda$ and $\boldsymbol{R}$

Because the free flow travel time of Link 1 equals one and Link 1 determines the value of $\varepsilon_{\max }$ in the current setting, $\sigma\left(T_{1}\right)$ and $\varepsilon_{\max }$ are numerically equal to each other. Hence, varying $\sigma\left(T_{1}\right)$ is equivalent to varying $\varepsilon_{\max }$. The minimum upper bound plotted in Figure 2(a) is strictly increasing in terms of $\sigma\left(T_{1}\right)$ (or $\varepsilon_{\max }$ ), and consistent with the result in Proposition 11. Moreover, it is convex in the range considered. Similarly, in Figure 2(b), the minimum upper bound is strictly increasing in terms of $\lambda$ and consistent with the result in Proposition 10. Furthermore, the curve seems to be straight. The curve in Figure 2(c) does accurately reflect the result in Proposition 8 that the minimum upper bound is linearly increasing in terms of $R$. The lower bound, on the other hand, is very close to each other, and is slowly decreasing in Figures 2a and b but is slowly increasing in Figure 2c. The gap between the lower bound and the corresponding minimum upper bound increases as the parameters get larger. Note that the figures only give the PoA of given instances (or the lower bounds), not the PoA of $I_{n, \varepsilon_{\max }}$. Hence, the loss of tightness of the minimum upper bound cannot be concluded by having a large gap observed."

The loss of tightness of $\rho_{R, \lambda}^{\min }$ is caused by the enlargement of the PoA in the derivation process. The detailed explanations are summarized in the following:

1) The numerator of the PoA, which is the TSTTB at RUE, is replaced by the upper bound of the TSTTB in Lemma 1. When the equality sign in (8) does not hold for every link and the equality sign in (9) does not hold for each pair of links, a larger value of $R$ leads to a larger difference in the two terms, a larger enlargement of the numerator, and eventually a looser minimum lower bound $\rho_{R, \lambda}^{\min }$. 
2) The denominator of the PoA, which is the TSTTB at RSO, is replaced by the lower bound of the TSTTB in Lemma 1 in the derivation process. The lower bound of the TSTTB at RSO is obtained by removing the safety margin in the TSTTB at RSO. If $R$ is larger, the reduction in the denominator of the PoA is larger, the enlargement on the PoA is larger, and $\rho_{R, \lambda}^{\min }$ is looser.

\section{Price of anarchy for the traditional user equilibrium network design problem}

The PoA has also been examined in tolling problems (e.g., Xiao et al., 2007; Han and Yang, 2008; Han et al., 2008b), a special case of NDPs according to the definition of Magnanti and Wong (1984). However, the PoA for traditional NDPs has not been studied. The optimal selection of link expansions in or link additions to an existing transportation network presents a traditional transportation NDP (e.g., Adbulaal and Leblanc, 1979; Yang and Bell, 1998; Szeto et al., 2014). There are other NDPs, such as turn restriction network design problems (Long et al., 2010; 2014), transit network design problems (e.g., Szeto and Wu, 2011; Szeto and Jiang, 2012, 2014), multimodal network design problems (e.g., Miandoabchi et al., 2012a,b), time-dependent network design problems (e.g., Szeto and Lo, 2008; Lo and Szeto, 2009; Szeto et al., 2010, 2015; Jiang and Szeto, 2015; Miandoabchi et al., 2015), and dynamic network design problems (Sun et al., 2014). A recent review is given by Farahani et al. (2013).

Both SO (e.g., Dantzig et al., 1979) and UE-NDPs (e.g., Abdulaal and Leblanc, 1979) have been addressed in the literature. Their main difference is that whereas the UE-NDP considers the selfish route choice behavior of travelers, the SO-NDP assumes that users are cooperative and choose paths to optimize the TSTT. In terms of model structure, compared with the SO-NDP, the UE-NDP includes UE constraints as extra constraints, which tightens the feasible solution set in general. Hence, the optimal TSTT obtained from the SO-NDP is always better than that obtained from the UE-NDP. To bound the proportion of TSTT increase due to the additional consideration of UE constraints in the NDP, a PoA for the UE-NDP is proposed in this section, one that is analogous to that found in UE traffic assignment.

Consider the link expansions in a traditional NDP. The capacity of a candidate link increases if the link is selected for improvement and remains unchanged if it is not selected. Note that this NDP can also consider link additions if the initial capacity of a candidate link is very close to zero. If the capacity of that link increases, then a link is effectively added to the network.

Recall that the capacity of a link $a$, i.e., $c_{a}$, can be captured by some of the coefficients as stated in Remark 1 after (7). Denote the link capacity increment or link capacity improvements as $\mathbf{y}=\left(y_{a}\right)_{a \in A}$. The total capacity of a link $a$ after design implementation becomes $\left(c_{a}+y_{a}\right)$ and alters some of the coefficients in the polynomial link travel time function. Denote the polynomial link travel time function after design implementation as $t_{a}\left(v_{a}, y_{a}\right)$. The feasible set of link expansions is denoted as

$$
\Omega_{\mathbf{y}}=\left\{\mathbf{y} \mid \sum_{a \in A} F_{a}\left(y_{a}\right) \leq B, 0 \leq y_{a} \leq u_{a}, a \in A\right\},
$$

in which $B$ is the total road construction budget (and is nonnegative). $F_{a}\left(y_{a}\right)$ is the construction cost for link $a$, and $u_{a}$ represents the maximum allowable expansion.

Based on the preceding notations, the SO-NDP can be formulated as follows: 


$$
\text { SO-NDP: } \min _{\mathbf{v} \in \Omega_{v}, y \in \Omega_{y}} \operatorname{TSTT}(\mathbf{v}, \mathbf{y})=\sum_{a \in A} t_{a}\left(v_{a}, y_{a}\right) v_{a},
$$

where $\operatorname{TSTT}(\mathbf{v}, \mathbf{y})$ denotes the TSTT at the link flow solution $\mathbf{v}$ and the link capacity increment $\mathbf{y}$. The solution set of program (77) is formed by linear constraints and hence is convex. It is also closed and non-empty. The objective function is continuous in terms of decision variables. Hence, a solution exists to the problem. Because the link travel time function and the solution set are convex in terms of link flows, the objective function is strictly convex in terms of link flows. If the function is also strictly convex in terms of link capacity improvements, then the solution is unique and also the corresponding TSTT. If not, the TSTT at all global minima must still be the same by definition.

The UE-NDP can be written as follows:

$$
\text { UE-NDP: } \min _{\mathbf{v} \in \Omega_{v}, \mathbf{y} \in \Omega_{y}} \operatorname{TSTT}(\mathbf{v}, \mathbf{y}) \text {, }
$$

subject to (2).

Following the discussion of the SO-NDP, it is clear that a solution exists to the problem. It is well known that the UE-NDP can be formulated into a bi-level non-convex minimization program (Yang and Bell, 1998) that may not have a unique solution.

Denote an optimal solution to the SO-NDP as a vector $\left(\mathbf{v}^{*}, \mathbf{y}^{*}\right)$, in which $\mathbf{y}^{*}=\left(y_{a}^{*}\right)_{a \in A}$ is an optimal link expansion vector and $\mathbf{v}^{*}=\left(v_{a}^{*}\right)_{a \in A}$ is an optimal link flow vector and a function of $\mathbf{y}^{*}$. The optimal TSTT to the SO-NDP can be represented as $\operatorname{TSTT}\left(\mathbf{v}^{*}\left(\mathbf{y}^{*}\right), \mathbf{y}^{*}\right)=\sum_{a \in A} t_{a}\left(v_{a}^{*}, y_{a}^{*}\right) v_{a}^{*}$. Moreover, denote $\left(\mathbf{v}^{R U E}\left(\mathbf{y}^{R U E}\right), \mathbf{y}^{R U E}\right) \quad$ and $\operatorname{TSTT}\left(\mathbf{v}^{R U E}\left(\mathbf{y}^{R U E}\right), \mathbf{y}^{R U E}\right)$ as an optimal solution to the UE-NDP and the corresponding TSTT, respectively. The PoA for a traditional UE-NDP can be defined as follows.

Definition 3. The PoA of a UE-NDP design instance $(G, \mathbf{D}, \mathbf{t}, B)$ is defined as

$$
\bar{\rho}(G, \mathbf{D}, \mathbf{t}, B)=\frac{\operatorname{TSTT}\left(\mathbf{v}^{U E}\left(\mathbf{y}^{U E}\right), \mathbf{y}^{U E}\right)}{\operatorname{TSTT}\left(\mathbf{v}^{*}\left(\mathbf{y}^{*}\right), \mathbf{y}^{*}\right)} .
$$

This PoA indicates how well the resources (e.g., the budget) have been allocated to improve system performance (i.e., the TSTT). An upper bound of $\bar{\rho}(G, \mathbf{D}, \mathbf{t}, B)$ is presented in the following theorem. Denote $\bar{I}_{n}$ as the set of all instances of the UE-NDP with polynomial travel time functions whose highest degree does not exceed $n$. Then, the PoA of $\bar{I}_{n}$ is $\bar{\rho}\left(\bar{I}_{n}\right)=\sup _{(G, \mathbf{D}, \mathbf{t}, B) \in \bar{I}_{n}} \bar{\rho}(G, \mathbf{D}, \mathbf{t}, B)$.

Theorem 3. The PoA of an instance $(G, \mathbf{D}, \mathbf{t}, B)$ for a traditional UE-NDP is bounded by

$$
\bar{\rho}(G, \mathbf{D}, \mathbf{t}, B) \leq \rho^{\prime}, \forall(G, \mathbf{D}, \mathbf{t}, B) \in \bar{I}_{n},
$$

in which $\rho^{\prime}$ is the upper bound of the PoA derived by Roughgarden (2005) for a traditional UE traffic assignment instance $(G, \mathbf{D}, \mathbf{t})$ that belongs to $I_{n}$.

Proof. Consider a network with the link capacity expansion set according to $\mathbf{y}^{*} \cdot \mathbf{v}^{*}\left(\mathbf{y}^{*}\right)$ and $\mathbf{v}^{U E}\left(\mathbf{y}^{*}\right)$ are the respective SO and UE link flow vectors obtained under this link 
expansion strategy. The theorem introduced by Roughgarden (2005) (see (5)) can be used to obtain

$$
\frac{\operatorname{TSTT}\left(\mathbf{v}^{U E}\left(\mathbf{y}^{*}\right), \mathbf{y}^{*}\right)}{\operatorname{TSTT}\left(\mathbf{v}^{*}\left(\mathbf{y}^{*}\right), \mathbf{y}^{*}\right)} \leq \rho^{\prime} .
$$

Moreover, $\left(\mathbf{v}^{U E}\left(\mathbf{y}^{*}\right), \mathbf{y}^{*}\right)$ is a feasible solution to the UE-NDP. Thus, the numerator of the left side of (81) is not less than the optimal objective value of the UE-NDP. Therefore, inequality (81) can be revised by replacing the numerator with $\operatorname{TSTT}\left(\mathbf{v}^{U E}\left(\mathbf{y}^{U E}\right), \mathbf{y}^{U E}\right)$ to obtain

$$
\frac{\operatorname{TSTT}\left(\mathbf{v}^{U E}\left(\mathbf{y}^{U E}\right), \mathbf{y}^{U E}\right)}{\operatorname{TSTT}\left(\mathbf{v}^{*}\left(\mathbf{y}^{*}\right), \mathbf{y}^{*}\right)} \leq \rho^{\prime} .
$$

The left side of (82) is $\bar{\rho}(G, \mathbf{D}, \mathbf{t}, B)$, and condition (82) holds for any network topology and demand pattern. Hence, the result follows. This completes the proof.

Remark 1: Theorem 3 is equivalent to $\bar{\rho}\left(\bar{I}_{n}\right) \leq \rho^{\prime}$. This theorem is applicable at any budget level. It is also applicable even when the travel time functions are not polynomial. In such case, $\rho^{\prime}$ should be replaced by the upper bound of the PoA for UE assignment with the travel time functions considered.

Remark 2: Theorem 3 is quite intuitive. Consider an unlimited budget. Then, the improved network has almost no congestion regardless of whether the improvement is based on the SO or UE design principle. (The travel time on each link almost equals its free flow travel time.) Consequently, the ratio $\bar{\rho}(G, \mathbf{D}, \mathbf{t}, B)$ is very close to one and must be smaller than an upper bound of the PoA of the instance $(G, \mathbf{D}, \mathbf{t})$ for a traditional UE traffic assignment problem, which is always larger than one. The latter is always bounded by the upper bound of the PoA of $I_{n}$ by definition. Consider another extreme case where the budget is zero. The SO- and UE-NDPs become SO and UE assignments, respectively. $\bar{\rho}(G, \mathbf{D}, \mathbf{t}, B)$ is bounded by the upper bound of the PoA for a traditional UE traffic assignment problem of the instance $(G, \mathbf{D}, \mathbf{t})$

Remark 3: In general, when the budget is larger, the resultant network is less congested (or the TSTT is lower) regardless of whether it is improved under the SO or UE design principle, but the two resultant TSTTs are closer than those before improvement. Hence, the PoA is smaller than that before the improvement and is closer to one.

\section{Price of anarchy for the reliability-based network design problem}

When confronted with network supply uncertainty, the system manager should consider the TSTT variation in the network design objective. When the TSTT variation is larger, a design that minimizes only the mean TSTT is not necessarily better than a design that minimizes both the mean TSTT and its variation. The mean TSTT may greatly underestimate the actual TSTT due to a large uncertainty. Therefore, the system manager should be risk averse and take extreme system performance into account. The current distribution-free reliability-based design model (e.g., $\mathrm{Ng}$ and Waller, 2009a) only considers the mean and standard deviation of the TSTT. In this paper, this consideration is relaxed via the addition of 
a multiplier to the standard deviation. This relaxation allows a more conservative design via a larger multiplier value. Thus, this section proposes using the TSTTB mentioned in Section 3.4 as the design objective.

Two types of NDPs are proposed: the RSO-NDP and the RUE-NDP. The RSO-NDP is analogous to the SO-NDP. It only has a different design objective and can be formulated into a single-level optimization program as follows:

$$
\text { RSO-NDP: } \min _{\mathbf{v} \in \Omega_{v}, \mathbf{y} \in \Omega_{y}} \operatorname{TSTTB}_{R}(\mathbf{v}, \mathbf{y}) \text {. }
$$

where $\operatorname{TSTTB}_{R}(\mathbf{v}, \mathbf{y})$ is the TSTTB at $(\mathbf{v}, \mathbf{y})$ given $R$. Note that $\operatorname{TSTTB}_{R}(\mathbf{v}, \mathbf{y})$ is not only a function of $\mathbf{v}$ but also a function of $\mathbf{y}$ because the travel time after improvement $t_{a}\left(v_{a}, y_{a}\right)$ is a function of both $v_{a}$ and $y_{a}$. If the selfish routing behavior of users is also considered, the RUE constraints mentioned in Section 3.3 must be incorporated into the design problem, resulting in the RUE-NDP, which can be formulated into a bi-level, non-linear, non-convex optimization program as follows:

$$
\begin{gathered}
\text { RUE-NDP: } \min _{\mathbf{v} \in \Omega_{v}, \mathbf{y} \in \Omega_{y}} \operatorname{TSTTB}_{R}(\mathbf{v}, \mathbf{y}), \\
\text { subject to (18). }
\end{gathered}
$$

Following the discussion in Sections 3.4, and 4, it is clear that a solution exists to the two problems, but multiple solutions are also possible.

Denote $\left(\mathbf{v}^{R S O}\left(\mathbf{y}^{R S O}\right), \mathbf{y}^{R S O}\right)$ and $\left(\mathbf{v}^{R U E}\left(\mathbf{y}^{R U E}\right), \mathbf{y}^{R U E}\right)$ as an optimal solution to the RSO-NDP and RUE-NDP, respectively. The PoA for the reliability-based NDP can be defined similar to that for the RUE and network design mentioned in Sections 3 and 4 as follows.

Definition 4. Given an RUE-NDP instance $(G, \mathbf{D}, \mathbf{t}, \sigma, B)$, and levels of risk aversion of system manager and travelers $R$ and $\lambda$, respectively, the PoA of this instance is defined as

$$
\bar{\rho}_{R, \lambda}(G, \mathbf{D}, \mathbf{t}, \boldsymbol{\sigma}, B)=\frac{\operatorname{TSTTB}_{R}\left(\mathbf{v}^{R U E}\left(\mathbf{y}^{R U E}\right), \mathbf{y}^{R U E}\right)}{\operatorname{TSTTB}_{R}\left(\mathbf{v}^{R S O}\left(\mathbf{y}^{R S O}\right), \mathbf{y}^{R S O}\right)} .
$$

Definition 5. Let $\bar{I}_{n, \varepsilon_{\max }}$ be the set of all RUE-NDP instances that satisfy the following conditions:

1) The link travel time functions are polynomial functions whose powers do not exceed the positive integer $n$;

2) The coefficients $\varepsilon_{a}, \forall a \in A$ (defined in Proposition 1) do not exceed the non-negative number $\varepsilon_{\max }$.

The PoA of $\bar{I}_{n, \varepsilon_{\max }}$ is

$$
\bar{\rho}_{R, \lambda}\left(\bar{I}_{n, \varepsilon_{\max }}\right)=\sup _{(G, \mathbf{D}, \mathbf{t}, \mathbf{\sigma}, B) \in \bar{I}_{n, \varepsilon_{\max }}} \bar{\rho}_{R, \lambda}(G, \mathbf{D}, \mathbf{t}, \boldsymbol{\sigma}, B) .
$$

Following the derivation process in Theorem 3, a set of upper bounds of $\bar{\rho}_{R}(G, \mathbf{D}, \mathbf{t}, \boldsymbol{\sigma}, B)$ can be obtained, which is stated below.

Theorem 4. The PoA of the RUE-NDP instance $(G, \mathbf{D}, \mathbf{t}, \boldsymbol{\sigma}, B)$ that belongs to $\bar{I}_{n, \varepsilon_{\max }}$ is 
bounded by the set of upper bounds of the PoA of the RUE traffic assignment instance $(G, \mathbf{D}, \mathbf{t}, \boldsymbol{\sigma})$ that belongs to $I_{n, \varepsilon_{\max }}$. That is,

$$
\begin{gathered}
\bar{\rho}_{R, \lambda}(G, \mathbf{D}, \mathbf{t}, \boldsymbol{\sigma}, B) \leq \frac{1+R \varepsilon_{\max }}{\Phi-n\left(\frac{\Phi\left(1+\lambda \varepsilon_{\max }\right)}{n+1}\right)^{\frac{n+1}{n}}}, \forall(G, \mathbf{D}, \mathbf{t}, \boldsymbol{\sigma}, B) \in \bar{I}_{n, \varepsilon_{\max }}, \\
\text { where } \Phi \in \Omega_{\Phi}=\left\{k \mid 0<k \leq \min \left(\frac{1}{1+\lambda \varepsilon_{\max }},\left(\frac{1}{n+\Delta}\right)^{n}\left(\frac{n+1}{1+\lambda \varepsilon_{\max }}\right)^{n+1}\right)\right\} .
\end{gathered}
$$

Similarly, $\bar{\rho}_{R, \lambda}\left(\bar{I}_{n, \varepsilon_{\max }}\right)$ is bounded by the right side of (86). Following the results of Theorems 3 and 4 , the following theorem can be concluded.

Theorem 5. The set of upper bounds of the PoA for the traditional UE-NDP and RUE-NDP are independent of the network topology $G$, demand $\mathbf{D}$, and budget $B$.

\section{Conclusion}

In this paper, an RSO assignment problem under supply uncertainty is proposed based on the concept of the TSTTB, and the existence and uniqueness of a solution to the problem are analyzed. The PoA of RUE traffic assignment with supply uncertainty is also defined. Based on the properties of the two reliability-based assignment problems, a set of upper bounds of the PoA is derived using polynomial link travel time functions and is proven to be independent of any network topology and demand pattern. The minimum upper bound is proven to be consistent with the upper bound of the PoA of traditional UE traffic assignment when network uncertainty is ignored by both the users and system manager or when no network uncertainty exists. The properties of the minimum upper bound in terms of the parameters of the RUE assignment problem are examined. The loss of tightness of the bound is also discussed.

A PoA is defined for the traditional UE-NDP with polynomial link travel time functions in which link expansion and addition are considered and the objective is to minimize the TSTT under budgetary and link expansion constraints. The PoA is proven not to exceed the upper bound of the PoA for traditional UE traffic assignment of the same instance, before any link expansion. This analysis is extended to the reliability-based NDPs. RUE- and RSO-NDPs are proposed with the objective of minimizing the TSTTB, and a PoA for the RUE-NDP is defined. The PoA is also proven not to exceed the set of upper bounds of the PoA for RUE traffic assignment of the same instance, before any link expansion.

The TSTTB is only one of the reasonable measures to define the RSO objective function, the PoA for RUE traffic assignment and network design. This measure considers the system manager's risk aversion perspective. An alternative measure is the sum of all individual travel-time-budget (TTB) that reflects users' perceived costs related to system uncertainty. This measure is from an economic perspective and is a counterpart of the stochastic-SO (SSO) concept under stochastic-UE (SUE). Similar to SSO, this "sum of individual TTB" would make the RSO objective function a path-based formulation, which may requires a totally different proof to derive the corresponding PoA. This proof is lengthy and hence is put in another paper. 


\section{Acknowledgements}

This research was jointly supported by grants (Nos. 201311159123 and 201411159063) from the University Research Committee of the University of Hong Kong and a grant from the National Natural Science Foundation of China (No. 71271183). The authors are grateful to the four reviewers for their useful comments.

\section{Appendix A: Uniqueness of RUE link flows and minimum travel time budgets}

Denote $\mathbf{f}^{\prime}$ and $\mathbf{f}^{\prime \prime}$ as two different RUE path flow solutions. $b_{p, r s}^{\prime}$ and $b_{p, r s}^{\prime \prime}$ are the travel time budgets associated with path $p$ and O-D pair rs corresponding to $\mathbf{f}^{\prime}$ and $\mathbf{f}^{\prime \prime}$, respectively. $v_{a}^{\prime}$ and $v_{a}^{\prime \prime}$ are the RUE flows on link $a$ corresponding to $\mathbf{f}^{\prime}$ and $\mathbf{f}^{\prime \prime}$, respectively. Based on Proposition 3, the followings are true:

$$
\begin{gathered}
\sum_{r s \in R S} \sum_{p \in P_{r s}}\left(f_{p, r s}^{\prime \prime}-f_{p, r s}^{\prime}\right) b_{p, r s}^{\prime} \geq 0, \text { and } \\
\sum_{r s \in R S} \sum_{p \in P_{r s}}\left(f_{p, r s}^{\prime}-f_{p, r s}^{\prime \prime}\right) b_{p, r s}^{\prime \prime} \geq 0 .
\end{gathered}
$$

Adding the above inequalities gives

$$
\sum_{r s \in R S} \sum_{p \in P_{r s}}\left(f_{p, r s}^{\prime}-f_{p, r s}^{\prime \prime}\right)\left(b_{p, r s}^{\prime \prime}-b_{p, r s}^{\prime}\right) \geq 0,
$$

which can be rewritten as

$$
\begin{aligned}
& \sum_{r s \in R S} \sum_{p \in P_{r s}}\left(f_{p, r s}^{\prime}-f_{p, r s}^{\prime \prime}\right)\left(\sum_{a \in A} t_{a}^{\prime \prime} \delta_{p, r s}^{a}-\sum_{a \in A} t_{a}^{\prime} \delta_{p, r s}^{a}\right)+ \\
& \sum_{r s \in R S} \sum_{p \in P_{r s}}\left(f_{p, r s}^{\prime}-f_{p, r s}^{\prime \prime}\right)\left(\lambda \sigma\left(T_{p, r s}^{\prime \prime}\right)-\lambda \sigma\left(T_{p, r s}^{\prime}\right)\right) \geq 0, \\
& \sum_{r s \in R S} \sum_{p \in P_{r s}}\left(\delta_{p, r s}^{a} f_{p, r s}^{\prime}-\delta_{p, r s}^{a} f_{p, r s}^{\prime \prime}\right)\left(\sum_{a \in A} t_{a}^{\prime \prime}-\sum_{a \in A} t_{a}^{\prime}\right) \\
& +\sum_{r s \in R S} \sum_{p \in P_{r s}}\left(f_{p, r s}^{\prime}-f_{p, r s}^{\prime \prime}\right)\left(\lambda \sigma\left(T_{p, r s}^{\prime \prime}\right)-\lambda \sigma\left(T_{p, r s}^{\prime}\right)\right) \geq 0 .
\end{aligned}
$$

Because of $\sigma\left(T_{p, r s}^{\prime}\right)=\sigma\left(T_{p, r s}^{\prime \prime}\right)$, the second summation term on the left side of (87) is zero. The first summation term can be expressed in terms of link flows due to $\sum_{r s \in R S} \sum_{p \in P_{r s}}\left(\delta_{p, r s}^{a} f_{p, r s}^{\prime}\right)=v_{a}^{\prime}$ and $\sum_{r s \in R S} \sum_{p \in P_{r s}}\left(\delta_{p, r s}^{a} f_{p, r s}^{\prime \prime}\right)=v_{a}^{\prime \prime}$. Hence, (87) can be reduced to

$$
\begin{gathered}
\sum_{a \in A}\left(v_{a}^{\prime \prime}-v_{a}^{\prime}\right)\left(t_{a}^{\prime}-t_{a}^{\prime \prime}\right) \geq 0 \text { or } \\
\sum_{a \in A}\left(v_{a}^{\prime \prime}-v_{a}^{\prime}\right)\left(t_{a}^{\prime \prime}-t_{a}^{\prime}\right) \leq 0 .
\end{gathered}
$$

According to the monotone and separable properties,

$$
\left(v_{a}^{\prime \prime}-v_{a}^{\prime}\right)\left(t_{a}^{\prime \prime}-t_{a}^{\prime}\right) \geq 0, \forall a \in A .
$$

Conditions (88) and (89) lead to

$$
\left(v_{a}^{\prime \prime}-v_{a}^{\prime}\right)\left(t_{a}^{\prime \prime}-t_{a}^{\prime}\right)=0, \forall a \in A .
$$

If the link performance function is strictly monotone with respect to its link flow, (90) implies $v_{a}^{\prime}=v_{a}^{\prime \prime}, \forall a \in A$, which further implies that the mean travel time on each link at RUE is unique and the minimum travel time budget for each OD pair is unique. 
If the link performance function is only monotone with respect to its link flow, (90) implies $t_{a}^{\prime \prime}=t_{a}^{\prime}, \forall a \in A$, and hence the minimum travel time budget for each OD pair is unique.

\section{Appendix B: The proof of Lemma 4}

Denote the left side of (44) as a function $\gamma_{a}\left(x_{a}\right)$ :

$$
\gamma_{a}\left(x_{a}\right)=k_{a m} x_{a}^{m} x_{a}-\Phi\left\{\left(1+\lambda \varepsilon_{a}\right) k_{a m} r_{a}^{m} x_{a}-k_{a m} r_{a}^{m} r_{a}\right\}, \quad \forall a \in A,
$$

which is continuous. The highest degree of $x_{a}$ in $\gamma_{a}\left(x_{a}\right)$ is $m+1$, which is always larger than or equal to two because $m \hat{\imath} \Phi_{+}$. Thus, the second derivative of $\gamma_{a}\left(x_{a}\right)$ in terms of $x_{a}$ is

$$
\frac{d^{2} \gamma_{a}\left(x_{a}\right)}{d x_{a}^{2}}=(m+1) m k_{a m} x_{a}^{m-1}, \forall a \in A,
$$

which is positive in $(0,+\infty)$ for all $m \hat{\imath} \Phi_{+}$. Thus, the function $\gamma_{a}\left(x_{a}\right)$ is a strictly convex function in terms of $x_{a}$ in the region of $(0,+\infty)$. The minimum value of $\gamma_{a}\left(x_{a}\right)$ in the region of $(0,+\infty)$ is attained at the stationary point $x_{a}^{*}$ where the first-order derivative of the objective function $\gamma_{a}\left(x_{a}\right)$ equals zero:

$$
\frac{d \gamma_{a}\left(x_{a}^{*}\right)}{d x_{a}}=0, \forall a \in A .
$$

Therefore,

$$
k_{a m}(m+1)\left(x_{a}^{*}\right)^{m}-k_{a m} \Phi\left(1+\lambda \varepsilon_{a}\right)\left(r_{a}\right)^{m}=0, \forall a \in A .
$$

The stationary point in the region of $(0,+\infty)$ is

$$
x_{a}^{*}=\left[\frac{\Phi\left(1+\lambda \varepsilon_{a}\right)}{m+1}\right]^{\frac{1}{m}} r_{a}, \forall a \in A .
$$

$x_{a}^{*}$ is always greater than zero if $r_{a}>0$ because both the numerator and denominator of the squared bracket term in (95) are positive. Thus, it can be concluded that the stationary point $x_{a}^{*}$ always exists in the region of $x_{a} \in(0,+\infty)$ if $r_{a}>0$. The minimum function value is

$$
\gamma_{a}\left(x_{a}^{*}\right)=\left[\Phi-m\left(\frac{\Phi\left(1+\lambda \varepsilon_{a}\right)}{m+1}\right)^{\frac{m+1}{m}}\right] k_{a m} r_{a}^{m} r_{a}, \forall a \in A .
$$

By definition, $\gamma_{a}\left(x_{a}\right) \geq \gamma_{a}\left(x_{a}^{*}\right), \forall a \in A$, which is equivalent to (44) in Lemma 4.

If $r_{a}=0$, then $\gamma_{a}\left(x_{a}\right)=k_{a m} x_{a}{ }^{m} x_{a} \geq 0$, which is a special case of (44). This completes the proof."

\section{Appendix C: The proof of Lemma 5}

Consider a relaxed function of $\theta\left(\Phi, m, \lambda, \varepsilon_{a}\right)$, denoted as $\theta^{\prime}\left(\Phi, m^{\prime}, \lambda, \varepsilon_{a}\right)$, where $m \mathbb{\Phi} \hat{i}+$ (i.e., $m \mathbb{\Phi}$ is a positive real number). Because $\theta^{\prime}\left(\Phi, m^{\prime}, \lambda, \varepsilon_{a}\right)$ is a continuous function of $m \Phi$, the derivative of $\theta^{\prime}\left(\Phi, m^{\prime}, \lambda, \varepsilon_{a}\right)$ can be taken in terms of $m \Phi$ to obtain 


$$
\frac{d}{d m^{\prime}} \theta^{\prime}\left(\Phi, m^{\prime}, \lambda, \varepsilon_{a}\right)=\frac{1}{m^{\prime}}\left(\frac{\Phi\left(1+\lambda \varepsilon_{a}\right)}{1+m^{\prime}}\right)^{\frac{m^{\prime}+1}{m^{\prime}}} \ln \left(\frac{\Phi\left(1+\lambda \varepsilon_{a}\right)}{1+m^{\prime}}\right), \forall a \in A
$$

The sign of $\frac{d}{d m^{\prime}} \theta^{\prime}\left(\Phi, m^{\prime}, \lambda, \varepsilon_{a}\right)$ depends solely on the sign of $\ln \left(\frac{\Phi\left(1+\lambda \varepsilon_{a}\right)}{1+m^{\prime}}\right)$ in the above expression because of $\frac{1}{m^{\prime}}\left(\frac{\Phi\left(1+\lambda \varepsilon_{a}\right)}{1+m^{\prime}}\right)^{\frac{m^{\prime}+1}{m^{\prime}}}>0$. Because $\Phi \leq \frac{1}{1+\lambda \varepsilon_{\max }}$ (see (43)), the right side of the above is not greater than $\frac{1}{1+\lambda \varepsilon_{a}}$ due to the definition of $\varepsilon_{\max }$. Hence,

$$
\Phi \leq \frac{1}{1+\lambda \varepsilon_{a}}, \forall a \in A
$$

Based on the above condition,

$$
\frac{\Phi\left(1+\lambda \varepsilon_{a}\right)}{1+m^{\prime}} \leq \frac{1}{1+m^{\prime}}<1 \text { and } \ln \left(\frac{\Phi\left(1+\lambda \varepsilon_{a}\right)}{1+m^{\prime}}\right)<0, \quad a \in A .
$$

Therefore, the sign of $\frac{d}{d m^{\prime}} \theta^{\prime}\left(\Phi, m^{\prime}, \lambda, \varepsilon_{a}\right)$ is negative, and $\theta^{\prime}\left(\Phi, m^{\prime}, \lambda, \varepsilon_{a}\right)$ is strictly decreasing in terms of $m \Phi$. The power $m \Phi$ can take a positive integer value, and hence $\theta\left(\Phi, m, \lambda, \varepsilon_{a}\right)$ is also decreasing in terms of $m \hat{\imath} \Phi_{+}$.

$$
\text { Furthermore, because } \begin{aligned}
\Phi & \leq\left(\frac{1}{m+\Delta}\right)\left(\frac{m+1}{1+\lambda \varepsilon_{\max }}\right)^{\frac{m+1}{m}} \text { (see (43)), } \\
& \Phi^{\frac{1}{m}} \leq\left(\frac{1}{m+\Delta}\right)\left(\frac{m+1}{1+\lambda \varepsilon_{a}}\right)^{\frac{m+1}{m}} .
\end{aligned}
$$

Due to $\left(\frac{1}{m+\Delta}\right)<\frac{1}{m}$, the right side of the above inequality is less than $\left(\frac{1}{m}\right)\left(\frac{m+1}{1+\lambda \varepsilon_{a}}\right)^{\frac{m+1}{m}}$, leading to:

$$
\Phi^{\frac{1}{m}}<\left(\frac{1}{m}\right)\left(\frac{m+1}{1+\lambda \varepsilon_{a}}\right)^{\frac{m+1}{m}}
$$

By multiplying both sides of the above inequality by a positive number $m\left(\frac{1+\lambda \varepsilon_{a}}{m+1}\right)^{\frac{m+1}{m}}$,

$$
\Phi^{\frac{1}{m}} m\left(\frac{1+\lambda \varepsilon_{a}}{m+1}\right)^{\frac{m+1}{m}}<m\left(\frac{1}{m}\right)\left(\frac{1+\lambda \varepsilon_{a}}{m+1}\right)^{\frac{m+1}{m}}\left(\frac{m+1}{1+\lambda \varepsilon_{a}}\right)^{\frac{m+1}{m}}=1 .
$$

It can then be concluded that 


$$
\Phi\left[1-\Phi^{\frac{1}{m}} m\left(\frac{1+\lambda \varepsilon_{a}}{m+1}\right)^{\frac{m+1}{m}}\right]=\Phi-m\left(\frac{\Phi\left(1+\lambda \varepsilon_{a}\right)}{m+1}\right)^{\frac{m+1}{m}}=\theta\left(\Phi, m, \lambda, \varepsilon_{a}\right)>0
$$

According to the above, $\theta\left(\Phi, m, \lambda, \varepsilon_{a}\right)$ is both strictly decreasing in terms of $m$ and positive. This completes the proof."

\section{Appendix D: The proof of Lemma 6}

According to Lemma 5, $\theta\left(\Phi, m, \lambda, \varepsilon_{a}\right)$ is a decreasing function in terms of $m$ when $\Phi \in \Omega_{\Phi}$. Thus,

$$
\theta\left(\Phi, 1, \lambda, \varepsilon_{a}\right) \geq \ldots \geq \theta\left(\Phi, n-1, \lambda, \varepsilon_{a}\right) \geq \theta\left(\Phi, n, \lambda, \varepsilon_{a}\right), \forall a \in A
$$

Based on the above relationship and condition (44) in Lemma 4, for any integer $m \in[1, n]$,

$$
k_{a m} x_{a}{ }^{m} x_{a}-\Phi\left\{\left(1+\lambda \varepsilon_{a}\right) k_{a m} r_{a}{ }^{m} x_{a}-k_{a m} r_{a}{ }^{m} r_{a}\right\} \geq \theta\left(\Phi, n, \lambda, \varepsilon_{a}\right) k_{a m} r_{a}{ }^{m} r_{a}, \quad \forall a \in A
$$

When $m=0$, the left side of (104) equals

$$
k_{a 0} x_{a}-\Phi\left\{\left(1+\lambda \varepsilon_{a}\right) k_{a 0} x_{a}-k_{a 0} r_{a}\right\}=\left(1-\Phi\left(1+\lambda \varepsilon_{a}\right)\right) k_{a 0} x_{a}+\Phi k_{a 0} r_{a}, \quad \forall a \in A .
$$

The term $\left(1-\Phi\left(1+\lambda \varepsilon_{a}\right)\right)$ in the right side of (105) is always non-negative because $\Phi \leq\left(1+\lambda \varepsilon_{a}\right)^{-1} \quad$ (i.e., $\Phi \in \Omega_{\Phi}$ ). The term $k_{a 0} x_{a}$ is also non-negative. Thus, the first term on the right side of (105) is non-negative. Hence, the right side of (105) is larger than or equal to the second term in the right side, leading to

$$
k_{a 0} X_{a}-\Phi\left\{\left(1+\lambda \varepsilon_{a}\right) k_{a 0} x_{a}-k_{a 0} r_{a}\right\} \geq \Phi k_{a 0} r_{a}, \forall a \in A .
$$

Clearly, from definition (45), $\Phi$ is not less than $\theta\left(\Phi, n, \lambda, \varepsilon_{a}\right)$ for any $n \hat{\imath} \Phi_{+}$. Hence, (104) also holds for $m=0$. Taking summation from $m=0$ to $n$, and replacing $\sum_{m=0}^{n} k_{a m} x_{a}{ }^{m}$ with $t_{a}\left(x_{a}\right)$, (104) becomes

$$
t_{a}\left(x_{a}\right) x_{a}-\Phi\left\{\left(1+\lambda \varepsilon_{a}\right) t_{a}\left(r_{a}\right) x_{a}-t_{a}\left(r_{a}\right) r_{a}\right\}=\theta\left(\Phi, n, \lambda, \varepsilon_{a}\right) t_{a}\left(r_{a}\right) r_{a}, \forall a \in A .
$$

After replacing $x_{a}$ with $v_{a}^{R S O}$ and $r_{a}$ with $v_{a}^{R U E}$, the inequality in Lemma 6 can be obtained. This completes the proof."

\section{References}

Aashtiani, H., 1979. The multi-modal traffic assignment problem. Ph.D. Thesis, Operations Research Center. MIT Press, Cambridge, MA.

Abdulaal, M., Leblanc, L.J., 1979. Continuous equilibrium network design models. Transportation Research Part B 13(1), 19-32.

Boyce, D.E., 1984. Urban transportation network equilibrium and design models: recent achievements and future prospective. Environment and Planning Part A 16(11), 1445-1474.

Chau, C.K., Sim, K.M., 2003. The price of anarchy for non-atomic congestion games with symmetric cost maps and elastic demands. Operations Research Letters 31(5), 327-334.

Chen, A., Kim, J., Zhou, Z., Chootinan, P., 2007. Alpha reliable network design problem. Transportation Research Record 2029, 49-57.

Chen, A., Zhou, Z., 2010. The $\alpha$-reliable mean-excess traffic equilibrium model with 
stochastic travel times. Transportation Research Part B 44(4), 493-513.

Correa, J., Schulz, A., Stier-Moses, N., 2004. Selfish routing in capacitated networks. Mathematics of Operations Research 29(4), 961-976.

Dantzig, G.B., Maier, S.F., Harvey, R.P., Lansdowne, Z.F., Robinson, D.W., 1979. Formulating and solving the network design problem by decomposition. Transportation Research Part B 13(1), 5-17.

Farahani, R.Z., Miandoabchi, E., Szeto, W.Y., Rashidi, H., 2013. A review of urban transportation network design problems. European Journal of Operational Research 229(2), 281-302.

Guo, X.L., Yang, H., Liu, T.L., 2010. Bounding the inefficiency of logit-based stochastic user equilibrium. European Journal of Operational Research 201(2), 463-469.

Han, D., Lo, H.K., 2002. A new alternating direction method for a class of nonlinear variational inequality problems. Journal of Optimization Theory and Applications 112(3), 549-560.

Han, D., Lo, H.K., Sun, J., Yang, H., 2008b. The toll effect on price of anarchy when costs are nonlinear and asymmetric. European Journal of Operational Research 186(1), 300-316.

Han, D., Lo, H.K., Yang, H., 2008a. On the price of anarchy for non-atomic congestion games under asymmetric cost maps and elastic demands. Computers \& Mathematics with Applications 56(10), 2737-2743.

Han, D., Yang, H., 2008. The multi-class, multi-criterion traffic equilibrium and the efficiency of congestion pricing. Transportation Research Part E 44(5), 753-773.

Jiang, Y. and Szeto, W.Y., 2015. Time-dependent transport network design that considers health cost. Transportmetrica A 11(1), 74-101.

Koutsoupias, E., Papadimitriou, C., 1999. Worst-case equilibria. In: Proceedings of the 16th Annual Symposium on Theoretical Aspects of Computer Science (STACS), Trier, Germany, Lecture Notes in Computer Science, vol. 1563, Springer, Berlin, 404-413.

Lam, W.H.K., Shao, H., Sumalee, A., 2008. Modeling impacts of adverse weather conditions on a road network with uncertainties in demand and supply. Transportation Research Part B 42(10), 890-910.

Liu, T.L., Huang, H.J., Yang, H., Guo, X.L., 2009. Equilibria and inefficiency in traffic networks with stochastic capacity and information provision. Transportation and Traffic Theory 2009: Golden Jubilee, 263-281.

Lo, H.K., Luo, X.W., Siu, B.W.Y., 2006. Degradable transport network: travel time budget of travelers with heterogeneous risk aversion. Transportation Research Part B 40(9), 792-806.

Lo, H.K., Szeto, W.Y., 2009. Time-dependent transport network design under cost-recovery. Transportation Research Part B 43(1), 142-158.

Long, J.C., Gao, Z.Y., Zhang, H.Z. and Szeto, W.Y., 2010. A turning restriction design problem in urban road networks. European Journal of Operational Research 206(3), 569-578.

Long, J.C., Szeto, W.Y. and Huang, H.J., 2014. A bi-objective turning restriction design problem in urban road networks. European Journal of Operational Research 237(2), 426-439.

Magnanti, T.L., Wong, R.T., 1984. Network design and transportation planning: Models and algorithms. Transportation Science 18(1), 1-55.

Meng, Q., Yang, H., 2002. Benefit distribution and equity in road network design. Transportation Research Part B 36(1), 19-35. 
Meng, Q., Yang, H., Bell, M.G.H., 2001. An equivalent continuously differentiable model and a locally convergent algorithm for the continuous network design problem. Transportation Research Part B 35(1), 83-105.

Miandoabchi, E., Daneshzand, F., Farahani, R.Z. and Szeto, W.Y., 2015. Time-dependent discrete road network design with both tactical and strategic decisions. Journal of the Operational Research Society 66, 894-913.

Miandoabchi, E., Farahani, R.Z. and Szeto, W.Y., 2012a. Bi-objective bimodal urban road network design using hybrid metaheuristics. Central European Journal of Operations Research 20(4), 583-621.

Miandoabchi, E., Farahani, R.Z., Dullaert, W. and Szeto, W.Y., 2012b. Hybrid evolutionary metaheuristics for concurrent multi-objective design of urban road and public transit networks. Networks and Spatial Economics 12(3), 441-480.

Nagurney, A., 1993. Network Economics: A Variational Inequality Approach. Kluwer Academic Publishers. Norwell, MA.

Ng, M.W., Waller, S.T., 2009a. Reliable system-optimal network design: convex mean-variance model with implicit chance constraints. Transportation Research Record 2090, 68-74.

Ng, M.W., Waller, S.T., 2009b. The evacuation optimal network design problem: model formulation and comparisons. Transportation Letters 1(2), 111-119.

Ordoñez, F., Stier-Moses, N., 2010. Wardrop equilibria with risk-averse users. Transportation Science 44(1), 63-86.

Perakis, G., 2004. The price of anarchy when costs are non-separable and asymmetric. Integer Programming and Combinatorial Optimization 3064, 46-58.

Roughgarden, T., 2003. The price of anarchy is independent of the network topology. Journal of Computer and System Sciences 67(2), 341-364.

Roughgarden, T., 2005. Selfish routing and price of anarchy. MIT Press. Cambridge, MA.

Roughgarden, T., Tardos, E., 2002. How bad is selfish routing? Journal of the ACM 49(2), 236-259.

Shao, H., Lam, W.H.K., Tam, M.L., 2006. A reliability-based stochastic traffic assignment model for network with multiple user classes under uncertainty in demand. Networks and Spatial Economics 6(3), 173-204.

Siu, B., Lo, H., 2008. Doubly uncertain transport network: degradable capacity and stochastic demand. European Journal of Operational Research 191(1), 166-181.

Smith, M.J., 1979. The existence, uniqueness and stability of traffic equilibria. Transportation Research Part B 13(4), 295-304.

Sumalee, A., Luathep, P., Lam, W.H.K., Connors, R.D., 2009. Transport network capacity evaluation and design under demand uncertainty. Transportation Research Record 2090, 17-28.

Sumalee, A., Watling, D.P., Nakayama, S., 2006. Reliable network design problem: case with uncertain demand and total travel time reliability. Transportation Research Record 1964, 81-90.

Sumalee, A., Xu, W., 2010. First-best marginal cost toll for a traffic network with stochastic demand. Transportation Research Part B 45(1), 41-59.

Sun, H., Gao, Z.Y., Long, J.C., Szeto, W.Y., 2014. A distributionally robust joint chance constrained optimization model for the dynamic network design problem under demand uncertainty. Networks and Spatial Economics 14(3-4), 409-433.

Szeto, W.Y. , Jiang, Y., 2012. Hybrid artificial bee colony algorithm for transit network design. Transportation Research Record 2284, 47-56.

Szeto, W.Y. and Wu, Y.Z., 2011. A simultaneous bus route design and frequency setting problem for Tin Shui Wai, Hong Kong. European Journal of Operational Research 209(2), 
141-155.

Szeto, W.Y., Jiang, Y., 2014. Transit route and frequency design: Bi-level modeling and hybrid artificial bee colony algorithm approach. Transportation Research Part B 67, 235-263.

Szeto, W.Y., Jiang, Y., Wang, D.Z.W. and Sumalee, A., 2015. A sustainable road network design problem with land use transportation interaction over time. Networks and Spatial Economics, DOI: 10.1007/s11067-013-9191-9.

Szeto, W.Y., Solayappan, M., Jiang, Y., 2011. Reliability-based transit assignment for congested stochastic transit networks. Computer-Aided Civil and Infrastructure Engineering 26(4), 311-326.

Szeto, W.Y., Lo, H.K., 2008. Time-dependent transport network improvement and tolling strategies. Transportation Research Part A 42(2), 376-391.

Szeto, W.Y., Jaber, X.Q. and O’Mahony, M., 2010. Time-dependent discrete network design frameworks considering land use. Computer-Aided Civil and Infrastructure Engineering 25(6), 411-426.

Szeto, W.Y., Wang, Y., Wong, S.C., 2014. The chemical reaction optimization approach to solving the environmentally sustainable network design problem. Computer-Aided Civil and Infrastructure Engineering 29(2), 140-158.

Uchida, T., Iida, Y., 1993. Risk assignment: a new traffic assignment model considering risk of travel time variation. In: Daganzo, C.F. (Ed.), Proceedings of the 12th International Symposium on Transportation and Traffic Theory. Elsevier. Amsterdam. pp. 89-105.

Wardrop, J.G., 1952. Some theoretical aspects of road traffic research. Proceedings of the Institute of Civil Engineers, Part II 1, 325-378.

Xiao, F., Yang, H., Han, D., 2007. Competition and efficiency of private toll roads. Transportation Research Part B 41(3), 292-308.

Yang, H., Bell, M.G.H., 1998. Models and algorithms for road network design: a review and some new developments. Transport Reviews 18(3), 257-278.

Yang, H., Han, D.R., Lo, H.K., 2008. Efficiency of atomic splittable selfish routing with polynomial cost functions. Networks and Spatial Economics 8(4), 443-451. 